Original article

\title{
Design, synthesis and bioevaluation of novel candidate selective estrogen receptor modulators
}

\author{
Yogesh Yadav ${ }^{\mathrm{a}, \mathrm{b}}$, Erin D. MacLean ${ }^{\mathrm{a}}$, Annyt Bhattacharyya ${ }^{\mathrm{a}}$, Virinder S. Parmar ${ }^{\mathrm{b}}$, Jan Balzarini ${ }^{\mathrm{c}}$, \\ Christopher J. Barden ${ }^{\mathrm{d}}$, Catherine K.L. Too ${ }^{\mathrm{e}}$, Amitabh Jha ${ }^{\mathrm{a}, *}$ \\ a Department of Chemistry, Acadia University, 6 University Avenue, Wolfville, NS B4P 2R6, Canada \\ ${ }^{\mathrm{b}}$ Bioorganic Laboratory, Department of Chemistry, University of Delhi, Delhi 110 007, India \\ ${ }^{\mathrm{c}}$ Rega Institute for Medical Research, Katholieke Universiteit Leuven, B-3000 Leuven, Belgium \\ ${ }^{\mathrm{d}}$ Cheminformatics and Drug Discovery Laboratory, IWK Health Centre, Halifax, NS B3K 6R8, Canada \\ e Department of Biochemistry \& Molecular Biology, Dalhousie University, Halifax, NS B3H 4R2, Canada
}

\section{A R T I C L E I N F O}

\section{Article history:}

Received 14 April 2011

Received in revised form

19 May 2011

Accepted 22 May 2011

Available online 30 May 2011

\section{Keywords:}

SERMs

Estrogen receptor

Breast cancer

Anticancer

Cytotoxic

Mannich bases

\begin{abstract}
A B S T R A C T
In an systematic attempt to develop novel Selective Estrogen Receptor Modulators (SERMs), chiral 1-((4-(2(dialkylamino)ethoxy)phenyl)(2-hydroxynaphthalen-1-yl)methyl)piperidin-4-ols were designed based on an accepted pharmacophore model. Simpler prototypes, viz. racemic 1-((2-hydroxynaphthalen-1-yl) arylmethyl)piperidin-4-ols, were first synthesized to develop kinetic resolution to pure enantiomers. Simultaneously, a series of racemic 1-((4-(2-(dialkylamino)ethoxy)phenyl)(2-hydroxynaphthalen-1-yl) methyl)piperidin-4-ols were evaluated against estrogen-responsive human MCF-7 breast cancer cells, but the compounds were found to be moderately active. The lack of potency could be due to the molecular bulk resulting in inadequate fit at the receptor. Subsequently, the molecular motif was modified to achiral 1-(4(2-(dialkylamino)ethoxy)benzyl)naphthalen-2-ols by removing the piperidinol moiety. Bioevaluation of this new series of compounds displayed significantly enhanced cytotoxicity against MCF-7 cells. A representative compound for this series showed estrogen receptor alpha binding activity and the action is that of an antagonist.
\end{abstract}

(c) 2011 Elsevier Masson SAS. All rights reserved.

\section{Introduction}

Selective Estrogen Receptor Modulators (SERMs) are chemical entities that alter the action of estrogen by binding to estrogen receptors $\left(\mathrm{ER}_{\alpha}\right.$ or $\mathrm{ER}_{\beta}$ subtypes) in cells [1]. In some cases, a SERM may antagonize the effects of estrogen, by preventing estrogen molecules from binding to the receptor sites. In other cases, a SERM may show estrogenic activity by mimicking estrogen. The pharmacodynamic response to a SERM is determined by both the position of the specific ER to which it binds, as well as the estrogen receptor subtype [2].

Current uses of SERMs include treatment of breast cancer and osteoporosis where estrogen receptors play an important role. Although most SERMs decrease the risk of breast cancer, the side effects of many of these drugs are deleterious [3]. For example, a drug may inhibit estrogen receptors in the breast, yet stimulate those in the uterus leading to increased risk of endometrial cancer.

\footnotetext{
* Corresponding author. Tel.: +1 902585 1515; fax: +1 9025851114 .

E-mail address: ajha@acadiau.ca (A. Jha).
}

These beneficial and harmful effects associated with a particular SERM must be carefully weighed to ensure an advantageous result [4]. Tamoxifen is currently the most widely used SERM and it inhibits the growth of estrogen receptor-positive breast cancer [2]. Tamoxifen can also be used to treat women who are currently healthy, yet who are at an increased risk for developing breast cancer [5]. Although tamoxifen is a benchmark in the treatment of estrogen-responsive breast cancer, its usage may be accompanied by negative side effects such as endometriosis [6]. Raloxifene, initially approved by the FDA in 1997 for the treatment of osteoporosis in postmenopausal women, is another SERM and it appears to have greater positive than negative effects. Clinical trials have shown raloxifene to be just as effective at preventing breast cancer as tamoxifen [6,7] and, importantly, the incidence of uterine cancer in these trials is substantially lower [6]. However, the observable side effects of raloxifene are thrombosis and fatal stroke in postmenopausal women [8].

The generic structure of the prototypical potential SERM designed for our study is shown in Fig. 1, along with structures of estradiol (the most potent estrogen) and raloxifene for comparison. It is evident that the designed molecules contain phenolic and 
<smiles>C[C@]12CCC3c4ccc(O)cc4CC[C@H]3[C@@H]1CC[C@@H]2O</smiles>

17-b-Estradiol<smiles>CCC(=C(c1ccccc1)c1ccccc1)c1ccc(OCCCN(C)C)cc1</smiles>

Tamoxifen<smiles>[R]N([R])CCOc1ccc(C(c2ccc(O)cc2O)N2CCC(O)CC2)cc1</smiles>

DesignedSERMs<smiles>[R20]OCc1cc(O)ccc1/C(C(=O)O)=C(\Sc1ccc(O)cc1)C(=O)c1ccc(OCCN2CCCCC2)cc1</smiles><smiles>Oc1ccc([C@H]2CCc3ccccc3[C@@H]2c2ccc(OCCCN3CCCC3)cc2)cc1</smiles>

Lasofoxifene

Fig. 1. Prototypical potential SERMs compared to 17- $\beta$-estradiol and several clinical SERMs.

alcoholic hydroxyl groups positioned appropriately to mimic estradiol. They also bear a dialkylaminoethoxyphenyl side-chain found in clinical SERMs (Fig. 1) [9]. Additionally, they have a chirality center making their topology non-planar like estrogen. It was hypothesized that these features will render potent selective estrogen receptor modulatory activity to the designed molecules.

A number of challenges are presented when dealing with the development of a drug containing a chirality center. Regulatory agencies have stopped approving racemic mixtures to be introduced in the market as drugs. It is a well-established fact that the desired biological response is due to one enantiomer only and that the other enantiomer, in nearly all cases, is either inactive or toxic [10]. Since our designed molecule has a chirality centre, we need either to synthesize it in an optically pure form or to resolve the enantiomeric pair into pure enantiomers.

To obtain the proof of concept in terms of biological activity and to develop a procedure to prepare the prototypical molecules in enantiomerically pure forms, two sets of experiments were simultaneously commissioned. First, a methodology was developed to produce a simplified version of designed molecules in optically pure form (Series 1, Fig. 2). Also, we synthesized the designed molecules devoid of the hydroxyl group at position 6 in racemic form (Series $\mathbf{2}$, Fig. 2) in parallel for bioevaluation to ascertain that they have the desired biological activity. Both series of molecules are easily synthesized in racemic form by performing a Mannich reaction between 2-naphthol, 4-piperidinol, and appropriate aromatic aldehydes. Although the presence of two $-\mathrm{OH}$ groups mimicking $17-\beta$-estradiol would have been ideal, we elected to synthesize series $\mathbf{2}$ for the following reasons: a) chemical syntheses of these analogs are short and less complicated, b) these compounds possess the complimentary 3D shape for the ER $\alpha$ binding pocket, and c) the lack of $-\mathrm{OH}$ groups mimicking 17 - $\beta$-estradiol is not considered detrimental as several clinical SERMs such as tamoxifen and lasofoxifene also lack one or both $-\mathrm{OH}$ groups (Fig. 1).

We recently published the synthesis of compounds $\mathbf{1 a}-\mathbf{j}$ and their enzyme-assisted kinetic resolution [10]. Herein, we report cytostatic activity of enantiomerically enriched compounds $\mathbf{1 a}-\mathbf{j}$ (Fig. 3) and their complementary monoacetate derivatives (Ac-1a-j; Fig. 3) against three anticancer cell lines, and synthesis of compounds $\mathbf{2 a}-\mathbf{f}$ and their cytotoxicity against estrogenresponsive MCF-7 breast cancer cell line. A new series of compounds (Series 3, Fig. 3) were designed based on the results obtained on series $\mathbf{2}$ compounds (vide infra) and their cytotoxicity was tested in MCF-7 cells.

\section{Chemistry}

Synthesis and enantiomeric enrichment of racemic Mannich bases $\mathbf{1 a}-\mathbf{j}$ with uncertain absolute stereochemistry and enantiomeric excess of resulting unreacted $(-) \mathbf{1} \mathbf{a}-\mathbf{h} \&(+) \mathbf{1} \mathbf{i}, \mathbf{j}$, and acetylated $(+) \mathbf{A c}-\mathbf{1} \mathbf{a}-\mathbf{h} \&(-) \mathbf{A c}-\mathbf{1 i}, \mathbf{j}$ has been described elsewhere [10].

Compounds of series $\mathbf{2}$ were synthesized in moderate yields by performing a Mannich reaction [10,11] between 2-naphthol, 4-piperidinol and appropriate 4-(2-(dialkylamino)ethoxy)benzaldehydes $(\mathbf{4 a}-\mathbf{f})$ in the presence of catalytic amounts of $p$-toluenesulfonic acid in a microwave reactor (Scheme 1 ). The products were purified by column chromatography and characterized by spectroscopic means. It should be noted that all compounds of this series are new to the chemical literature and they displayed molecular dynamics (typical of numerous piperidine containing compounds [11]) in their ${ }^{1} \mathrm{H}$ and ${ }^{13} \mathrm{C}$ NMR spectra.

Synthesis of compounds of series $\mathbf{3}$ is depicted in Scheme 2. 4-(Dialkylaminoethoxy)benzaldehydes $(\mathbf{4 a}-\mathbf{h})$, synthesized from commercially available dialkylaminoethyl chloride (Scheme 1) [12] or from 4-hydroxybenzaldehyde, dibromoethane and dialkylamine (Scheme 2) [13], were reduced [14] to the corresponding benzyl alcohol 5a-h. Subsequent benzylation reaction [15] of 2-naphthol with these benzyl alcohols in the presence of boron trifluoride etherate resulted in formation of the desired 1-(4-(2-(dialkylamino) ethoxy)benzyl)naphthalen-2-ols (3a-h; Scheme 2). Compounds $\mathbf{3 a}-\mathbf{h}$, hitherto unknown in chemical literature, were unambiguously characterized by spectroscopic means. These compounds

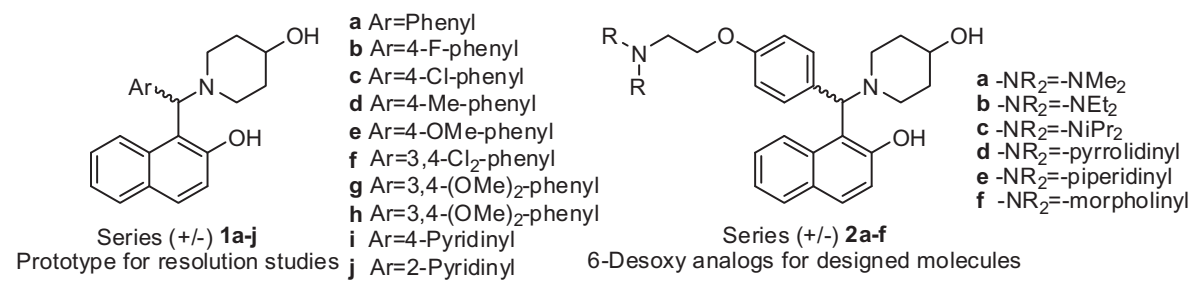

Fig. 2. Racemic compounds for preliminary bioevaluation and resolution studies. 


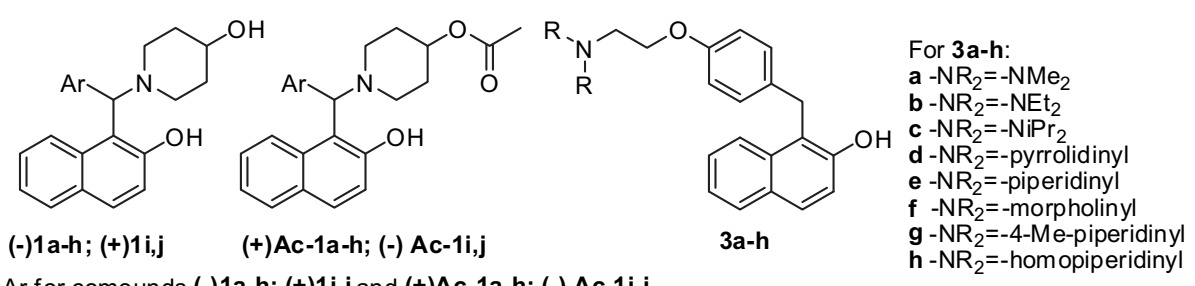

Ar for comounds (-)1a-h; (+)1i,j and (+)Ac-1a-h; (-) Ac-1 i,j

are the same as indicated in Figure 2.

Absolute stereochemistry unknown.

Fig. 3. Molecular structures of compounds bioevaluated.

were subsequently converted to corresponding water-soluble $\mathrm{HCl}$ salts by passing $\mathrm{HCl}$ gas to their ethereal solution. The vacuum-dried solid precipitate was used for MCF-7 cytotoxicity assays.

\section{Biological results and discussion}

Mannich bases are known to possess anticancer activity which is attributed to their ability to deaminate under physiological conditions and to form an electrophilic reactive intermediate capable of alkylation of the cellular nucleophiles [16,17]. Enantiomerically enriched compounds $(-) \mathbf{1} \mathbf{a}-\mathbf{h},(+) \mathbf{1} \mathbf{i}, \mathbf{j},(+) \mathbf{A c}-\mathbf{1 a}-\mathbf{h}$ and $(-)$ Ac-1i,j were subjected to cytostatic evaluation against murine leukemic L1210 and human lymphoblast Molt4/C8 and CEM cell lines [18]. Melphalan was used as the reference drug. The results are presented in Table 1.

As evident from the table, all tested compounds are inferior to melphalan as a cytotoxin against selected tumor cell lines. However, most of them are moderately cytostatic with their $\mathrm{IC}_{50}$ in the middle micromolar range. Compounds $(-) \mathbf{1 e}-\mathbf{g}$ and their acetylated counterparts $(+) \mathbf{A c}-\mathbf{1 e}-\mathbf{g}$ were the two cytostatic agents with lowest average $\mathrm{IC}_{50}$ values. Pyridine-containing analogs displayed the least cytostatic activity in the series.

The main focus of this investigation was on compounds of series 2. Compounds $\mathbf{2 a}-\mathbf{f}$ were evaluated against estrogen-responsive MCF-7 breast cancer cells. The $\mathrm{IC}_{50}$ values are presented in Table 2. The cytotoxicity potential of most compounds of series 2 were found to be comparable to that of tamoxifen and were not considered an improvement. This incited us to reconsider our design in terms of the fit of prototypical molecules in the ER $\alpha$ ligand binding region. Based on the reported binding of estradiol [9] and raloxifene [20] in the ER $\alpha$ ligand binding domain, it is expected that the tertiary amine will bind with Asp 351 whereas the hydroxy group of the piperidinol ring will associate with His 524. However, when the skeleton of compounds $\mathbf{2 a}-\mathbf{f}$ was compared with that of estradiol, superimposing the tetralin ring of estradiol with the naphthalene ring of the compounds of series 2 , it became apparent that the hydroxyl group on piperidinol did not align with the estradiol aliphatic hydroxyl group. To accurately ascertain this, we decided to perform docking studies on the two enantiomers of $\mathbf{2 b}$, a representative compound from series $\mathbf{2}$, in the ER $\alpha$ ligand binding domain. The results of the docking studies indicated that while both enantiomers of $\mathbf{2 b}$ were able to fit in the ER $\alpha$ ligand binding domain, the $R$-enantiomer showed relatively better binding than the $S$-enantiomer. This may be responsible for the relatively higher MCF-7 IC 50 value for the racemic mixture.

Our analysis suggested that in order to have tighter binding of the ligands with the ER $\alpha$ ligand binding domain, the position of the aliphatic hydroxyl has to be modified and this avenue is currently being investigated in our laboratory. We envisioned that the predicament caused by the current location of 4-piperidinol ring leading to less than optimal binding with the ER $\alpha$ site (at least in one enantiomer) can easily be solved by removing the piperidinol ring altogether. Also, this will make the compounds achiral which eliminates the requirement of optical resolution. Although this exercise will lead to the loss of a potential H-bond donor/acceptor system, it may not be detrimental to the desired biological activity (clinical SERM tamoxifen does not possess any hydroxyl group; Fig. 2). To gain confidence and to verify this argument, we docked the analog of compound $\mathbf{2 b}$ devoid of the piperidinol ring (3b, Fig. 3 ) in the ER $\alpha$ ligand binding domain and found that it indeed showed a tighter and comfortable binding. Fig. 4 clearly highlights the interaction of the tertiary amine with Asp 351. The naphthalene ring nicely fits in the hydrophobic pocket with arene-H interactions of Phe 404 and Leu 387.

The new scaffold resulting from the loss of 4-piperidinol moiety from compounds of series 2 constitute compounds of series 3. Synthesis of this series was possible by reductive deamination [21] of compounds of series $\mathbf{2}$ but owing to relatively lower yields of series $\mathbf{2}$ compounds, we elected to synthesize series $\mathbf{3}$ compounds directly by $\mathrm{C}-1$ benzylation of 2-naphthol [15]. The cytotoxicity results, shown in Table 2, clearly established compounds of series 3 as important leads toward the development of future SERMs. All compounds except 3f displayed 3-6 fold improvement over tamoxifen in the MCF-7 cytotoxicity assay. Compound 3f with morpholine in the basic side-chain displayed moderate cytotoxicity values with $\mathrm{IC}_{50}$ 's in the range of $25-50 \mu \mathrm{M}$. This is a significant improvement over $\mathrm{IC}_{50}$ of $423.7 \mu \mathrm{M}$ for $\mathbf{2 f}$, the morpholine analog from series $\mathbf{2}$. The most potent cytotoxic compound in series $\mathbf{3}$ is $\mathbf{3 g}$, which contains a 4-methylpiperidine ring in the basic side-chain.

We next determined the ability of the most potent candidate $\mathbf{3 g}$ to bind and antagonize human ER $\alpha$ using a commercial kit [23]. The results of this colorimetric assay are displayed in Fig. 4. The agonist

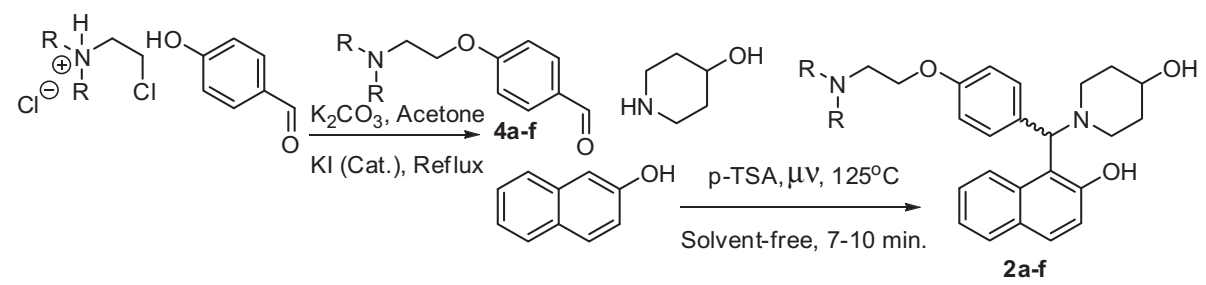

Scheme 1. Synthetic scheme adopted to prepare compounds of series $\mathbf{2}$. The $-\mathrm{NR}_{2}$ groups for compounds $\mathbf{2 a}-\mathbf{f}$ are indicated in Fig. 2 . 


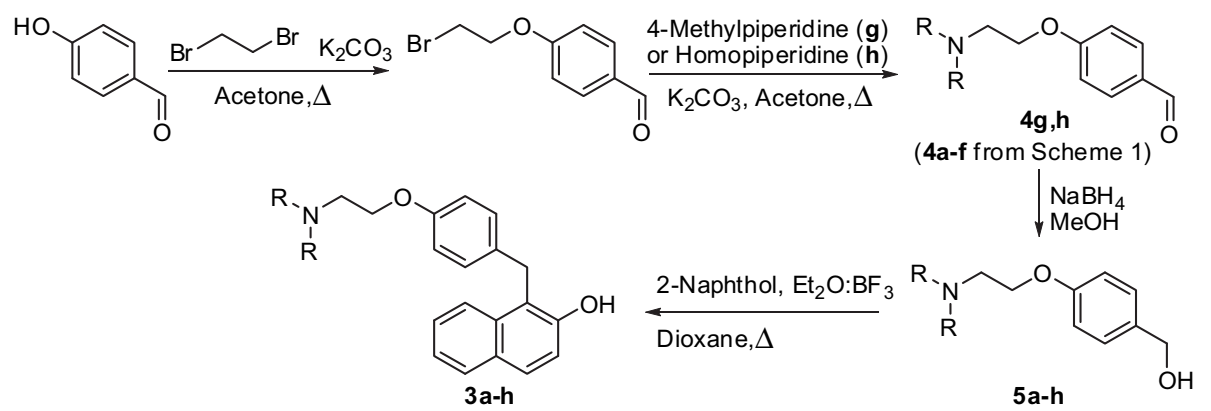

Scheme 2. Synthetic scheme adapted to prepare compounds of series $\mathbf{3}$. The $-\mathrm{NR}_{2}$ groups for compounds $\mathbf{3 a}-\mathbf{h}$ are indicated in Fig. 2 and Table 2 .

estradiol (E2) and antagonist tamoxifen (TEM) controls were used at $25 \mu \mathrm{M}$ whereas the test compound $\mathbf{3 g}$ was used at concentrations of 25 and $2.5 \mu \mathrm{M}$. Fig. 5 clearly shows that compound $\mathbf{3 g}$ is an antagonist at the concentrations used and is a considerably more potent ER binder and antagonist than tamoxifen.

\section{Conclusion}

In conclusion, our iterative study which began with design, synthesis and bioevaluation of 1-((4-(2-(dialkylamino)ethoxy) phenyl)(2-hydroxynaphthalen-1-yl)methyl)piperidin-4-ols as potential SERMs has led to the identification of simpler 1-(4-(2-(dialkylamino)ethoxy)benzyl)-naphthalen-2-ols as lead prototypes for further development. These compounds displayed superior cytotoxicity toward estrogen-responsive human MCF-7 breast cancer cells than tamoxifen. A representative compound for the series has also shown significant binding and antagonistic effects against human ER $\alpha$ in an in-vitro ELISA assay. We are actively pursuing modification on this motif to improve ER $\alpha$-dependent breast cancer cytotoxicity as well as ER $\alpha$ antagonistic activity which will translate into in-vivo efficacy in breast cancer animal models.

\section{Experimental section}

\subsection{Chemistry}

\subsubsection{General}

Melting points were determined on a MEL-TEMP II apparatus. The IR spectra were recorded on a Nicolet AVATAR 300 FT-IR instrument by making $\mathrm{KBr}$ discs for solid samples and thin films for oils. The ${ }^{1} \mathrm{H}$ NMR and ${ }^{13} \mathrm{C}$ NMR spectra were recorded on a Bruker AC-300 Avance spectrometer or Bruker ARX-300 spectrometer at 300 and at $75.5 \mathrm{MHz}$, respectively. The chemical shift values are on $\delta$ scale and the coupling constants $(J)$ are in Hz. The
HRMS were recorded in positive ion mode on an Ion Spec Fourier transform mass spectrometer. CEM Discover S-class microwave reactor was used for the synthesis of compounds. All chemicals used were purchased either from Aldrich Chemical Co. or Fluka Chemicals Co., Canada and used without further purification. Analytical TLCs were performed on pre-coated Merck silica gel 60F254 plates; the spots were detected under UV light. Silica gel (100-200 mesh) was used for column chromatography. After column chromatography, fraction containing products were evaporated under reduced pressure and dried $12 \mathrm{~h}$ under high vacuum to give the product otherwise specified. Some reactions were conducted under an atmosphere of nitrogen when anhydrous solvents were used.

\subsubsection{General procedure for the synthesis of 4-(dialkylaminoethoxy)- benzaldehydes $\mathbf{4 a}-\boldsymbol{f}$}

In a dried single-neck round bottom flask, 4-hydroxy-l benzaldehyde ( $40 \mathrm{mmol}$ ), potassium carbonate $(122 \mathrm{mmol}$ ) and dry acetone $(160 \mathrm{ml})$ were taken and the contents refluxed for $2 \mathrm{~h}$. The reaction mixture was brought to room temperature and catalytic amount of potassium iodide was added, followed by the gradual addition of appropriate dialkylaminoethyl chloride hydrochloride $(45 \mathrm{mmol})$ dissolved in dry acetone $(50 \mathrm{ml})$ through a pressure-equalizing addition funnel and the reaction mixture was allowed to reflux again. The contents were regularly monitored for reaction progress by TLC using $10 \%$ methanol/dichloromethane as the solvent system. The reaction was generally complete in 10-13 h. At this point, reaction mixture was filtered under suction and the solid inorganic salts were washed with acetone $(3 \times 60 \mathrm{ml})$. The solvent was evaporated under reduced pressure and the residue was purified by column chromatography over silica gel (6-8\% methanol in dichloromethane, $\mathrm{v} / \mathrm{v}$ as eluent) to afford the pure aldehydes $\mathbf{4 a}-\mathbf{f}$ as colorless oils in $49-92 \%$ yields. The structures of $\mathbf{4 a}-\mathbf{f}$ were unambiguously established from the

Table 1

Cytostatic potency of compounds (-)1a-h; (+)1i,j and (+)Ac-1a-h; $(-)$ Ac-1i,j against murine L1210, and human Molt4/C8 and CEM T-lymphocyte cell lines.

\begin{tabular}{|c|c|c|c|c|c|c|c|}
\hline \multirow[t]{2}{*}{ Ar } & & \multicolumn{3}{|c|}{$(-) \mathbf{1} \mathbf{a}-\mathbf{h} ;(+) \mathbf{1} \mathbf{i}, \mathbf{j} I C_{50}(\mu \mathrm{M})$} & \multicolumn{3}{|c|}{$(+) \mathbf{A c}-\mathbf{1 a}-\mathbf{h} ;(-) \mathbf{A c}-\mathbf{1 i}, \mathbf{j} I \mathrm{IC}_{50}(\mu \mathrm{M})$} \\
\hline & & L1210 & Molt4/C8 & CEM & L1210 & Molt4/C8 & CEM \\
\hline $\mathbf{a}$ & Phenyl & $50 \pm 1$ & $43 \pm 4$ & $36 \pm 6$ & $58 \pm 17$ & $38 \pm 8$ & $31 \pm 14$ \\
\hline b & 4-F-phenyl & $44 \pm 0$ & $41 \pm 3$ & $24 \pm 2$ & $45 \pm 2$ & $40 \pm 4$ & $27 \pm 1$ \\
\hline c & 4-Cl-phenyl & $46 \pm 2$ & $33 \pm 7$ & $19 \pm 4$ & $41 \pm 3$ & $35 \pm 5$ & $28 \pm 2$ \\
\hline d & 4-Me-phenyl & $44 \pm 1$ & $39 \pm 6$ & $29 \pm 2$ & $45 \pm 2$ & $47 \pm 8$ & $35 \pm 7$ \\
\hline e & 4-MeO-phenyl & $42 \pm 2$ & $35 \pm 7$ & $14 \pm 1$ & $43 \pm 2$ & $42 \pm 5$ & $22 \pm 0$ \\
\hline f & 3,4- $\mathrm{Cl}_{2}$-phenyl & $43 \pm 0$ & $36 \pm 2$ & $17 \pm 3$ & $31 \pm 1$ & $34 \pm 3$ & $15 \pm 1$ \\
\hline g & 3,4-(OMe) $)_{2}$-phenyl & $50 \pm 3$ & $18 \pm 3$ & $25 \pm 7$ & $48 \pm 2$ & $12 \pm 2$ & $24 \pm 8$ \\
\hline h & 3,4-( $\left.\mathrm{OCH}_{2} \mathrm{O}\right)$-phenyl & $45 \pm 1$ & $44 \pm 4$ & $37 \pm 7$ & $48 \pm 3$ & $69 \pm 10$ & $51 \pm 4$ \\
\hline i & 4-Pyridyl & $154 \pm 23$ & $107 \pm 65$ & $133 \pm 44$ & $80 \pm 5$ & $59 \pm 1$ & $79 \pm 0$ \\
\hline \multirow[t]{2}{*}{$\mathbf{j}$} & 2-Pyridyl & $118 \pm 34$ & $52 \pm 14$ & $54 \pm 21$ & $92 \pm 10$ & $49 \pm 10$ & $55 \pm 1$ \\
\hline & Melphalan ${ }^{\mathrm{a}}$ & $2.13 \pm 0.03$ & $3.24 \pm 0.79$ & $2.47 \pm 0.03$ & & & \\
\hline
\end{tabular}

\footnotetext{
${ }^{a}$ The data for melphalan are reproduced from Dimmock et al. Eur. J. Med. Chem. 35 (2000) 967-977 [19].
} 
Table 2

In-vitro cytotoxicity data of compounds of series $\mathbf{2}$ and $\mathbf{3}$ against MCF-7 breast cancer cells

\begin{tabular}{|c|c|c|}
\hline \multirow[t]{2}{*}{ Compound ID } & \multicolumn{2}{|c|}{$\mathrm{IC}_{50}(\mu \mathrm{M})$ against MCF-7 } \\
\hline & Series 2 & Series 3 \\
\hline $\mathbf{a}$ & 22.6 & 10.18 \\
\hline b & 19.0 & 6.20 \\
\hline c & 52.3 & 7.54 \\
\hline d & 37.5 & 6.71 \\
\hline $\mathbf{e}$ & 36.4 & 6.82 \\
\hline f & 423.7 & $>25 ;<50$ \\
\hline g & - & 4.38 \\
\hline $\begin{array}{l}\text { h } \\
\text { Tamoxifen }\end{array}$ & $-\overline{31.0}$ & 6.21 \\
\hline
\end{tabular}

a The data for tamoxifen is reproduced from Seeger et al. J. Steroid Biochem. Mol. Biol. 84 (2003) 255-257 [22].

comparison of their spectral data with reported values [13,24,25]. The spectroscopic data of compound $\mathbf{4 b}$ which is not reported in literature is being included here.

5.1.2.1. 4-(Diethylaminoethoxy)benzaldehyde (4b). Colorless oil, yield 92\%. UV (EtOH) $\lambda_{\max }: 224,273$ and $319 \mathrm{~nm}$. IR (nujol): 2966, 2872, 2826, 1693, 1604, 1509, 1466, 1361,1260, 1185 1158, 1016 and $832 \mathrm{~cm}^{-1} .{ }^{1} \mathrm{H} \mathrm{NMR}\left(300 \mathrm{MHz}, \mathrm{CDCl}_{3}\right): \delta 1.06(6 \mathrm{H}, \mathrm{t}, J=7.2 \mathrm{~Hz}, 2 \times$ $\left.\mathrm{CH}_{3}\right), 2.64\left(4 \mathrm{H}, \mathrm{q}, J=7.2 \mathrm{~Hz}, 2 \times \mathrm{NCH}_{2}\right), 2.89(2 \mathrm{H}, \mathrm{t}, J=6.0 \mathrm{~Hz}$, $\left.-\mathrm{NCH}_{2}\right), 4.11\left(2 \mathrm{H}, \mathrm{t}, J=6.3 \mathrm{~Hz}, \mathrm{OCH}_{2}\right), 7.01(2 \mathrm{H}, \mathrm{d}, J=8.7 \mathrm{~Hz}, 2 \times$ $\mathrm{Ar}-\mathrm{H}), 7.83(2 \mathrm{H}, \mathrm{d}, J=8.7 \mathrm{~Hz}, 2 \times \mathrm{Ar}-\mathrm{H}), 9.87(1 \mathrm{H}, \mathrm{s}, \mathrm{CHO}) .{ }^{13} \mathrm{C} \mathrm{NMR}$ (75.5 MHz, $\left.\mathrm{CDCl}_{3}\right): \delta 11.85\left(2 \times \mathrm{CH}_{3}\right), 47.89\left(2 \times \mathrm{NCH}_{2}\right), 51.57\left(\mathrm{NCH}_{2}\right)$, $67.12\left(\mathrm{OCH}_{2}\right), 114.83$ (C-2 \& C-6), 129.92 (C-1), 131.93 (C-3 \& C-5), $163.96(\mathrm{C}-4), 190.74(\mathrm{C}=\mathrm{O})$. HRMS $\mathrm{m} / z$ calculated for $\mathrm{C}_{13} \mathrm{H}_{19} \mathrm{NO}_{2}$ $[\mathrm{M}+\mathrm{H}]^{+}$222.1416, observed $[\mathrm{M}+\mathrm{H}]^{+} 222.1411$.

\subsubsection{General procedure for the synthesis of compounds $\mathbf{2 a}-\boldsymbol{f}$}

A mixture of 2-naphthol (12 mmol), 4-hydroxypiperidine (12 mmol), appropriate (dialkylamino)ethoxybenzaldehydes $(10 \mathrm{mmol})$ and catalytic amount of $p$-TSA $(50 \mathrm{mg})$ were irradiated in
Discover S-Class CEM microwave oven for $7-10 \mathrm{~min}$. $(2.5 \mathrm{~min} \times 3$ or 4 , to avoid overheating). The reaction mixture was cooled and purified by column chromatography over silica gel using methanoldichloromethane $(8-10 \%)$ as eluent to afford the pure compounds $\mathbf{2 a}-\mathbf{f}$ in $38-47 \%$ yields. The structures of compounds $\mathbf{2 a}-\mathbf{f}$ were unambiguously established from the analysis of their spectral data (IR, ${ }^{1} \mathrm{H},{ }^{13} \mathrm{C}$ NMR and mass spectra). It should be noted that the peak for the two $-\mathrm{NCH}_{2}$ carbons of the 4-hydroxypiperidine ring were consistently not seen at room temperature due to the molecular dynamics as mentioned in the Results and Discussion section.

5.1.3.1. 1-[[4-(2-(Dimethylamino)ethoxy)phenyl](2-hydroxynaphthalen-1-yl)methyl] piperidin-4-ol (2a). Light yellow solid, yield 38\%, m.p. $86-88^{\circ} \mathrm{C}$. UV(EtOH) $\lambda_{\max }: 231,280$ and $334 \mathrm{~nm}$. IR (KBr): 3393 , 2936, 2801, 1608, 1511, 1455, 1367, 1240, 1167, 1053, 1011, 946, 832, and $816 \mathrm{~cm}^{-1} .{ }^{1} \mathrm{H}$ NMR $\left(300 \mathrm{MHz}, \mathrm{CDCl}_{3}\right) \delta 1.52-1.64\left(2 \mathrm{H}, \mathrm{m}, \mathrm{CH}_{2}\right)$, $1.81-1.89\left(2 \mathrm{H}, \mathrm{m}, \mathrm{CH}_{2}\right), 2.30\left(6 \mathrm{H}, \mathrm{s}, 2 \times \mathrm{NCH}_{3}\right), 2.35\left(2 \mathrm{H}, \mathrm{brs}, \mathrm{NCH}_{2}\right)$, 2.66-2.70 $\left(4 \mathrm{H}, \mathrm{m}, 2 \times \mathrm{NCH}_{2}\right), 3.72(1 \mathrm{H}, \mathrm{brs}, \mathrm{OCH}), 3.99(2 \mathrm{H}, \mathrm{t}$, $\left.J=5.7 \mathrm{~Hz}, \mathrm{OCH}_{2}\right), 5.17$ (1H, brs, $\left.\mathrm{Ar}_{2} \mathrm{CHN}\right), 6.80-6.83(2 \mathrm{H}, \mathrm{m}, 2 \times$ $\mathrm{Ar}-\mathrm{H}), 7.14-7.28(6 \mathrm{H}, \mathrm{m}, 6 \times \mathrm{Ar}-\mathrm{H}), 7.65-7.68(2 \mathrm{H}, \mathrm{m}, 2 \times \mathrm{Ar}-\mathrm{H})$. ${ }^{13} \mathrm{C}$ NMR (75.5 MHz, $\left.\mathrm{CDCl}_{3}\right): \delta 28.51\left(2 \times \mathrm{CH}_{2}\right), 46.12\left(2 \times \mathrm{NCH}_{3}\right)$, $58.49\left(\mathrm{NCH}_{2}\right), 66.13\left(\mathrm{OCH}_{2}\right), 69.85(\mathrm{OCH}), 71.20\left(\mathrm{Ar}_{2} \mathrm{CHN}\right), 115.18$, 116.65, 120.30, 121.47, 122.81, 126.80, 127.25, 129.10, 129.27, 129.74, $130.56,132.20,132.64,155.66 \& 158.83(\mathrm{Ar})$. HRMS $m / z$ calculated for $\mathrm{C}_{26} \mathrm{H}_{32} \mathrm{~N}_{2} \mathrm{O}_{3}[\mathrm{M}+\mathrm{H}]^{+} 421.2413$, observed $[\mathrm{M}+\mathrm{H}]^{+} 421.2464$.

5.1.3.2. 1-[[4-(2-(Diethylamino)ethoxy)phenyl](2-hydroxynaphthalen-1-yl)methyl] piperidin-4-ol (2b). Light brown solid, yield 41\%, m.p. $64-66{ }^{\circ} \mathrm{C}$. UV(EtOH) $\lambda_{\max }$ : 232, 280 and $337 \mathrm{~nm}$. IR (KBr): $3408,2967,2932,2850,1609,1511,1453,1304,1239,1178,1053,945$ and $832 \mathrm{~cm}^{-1} .{ }^{1} \mathrm{H}$ NMR (300 MHz, $\left.\mathrm{CDCl}_{3}\right)$ : 0.99-1.07 (6H, m, $2 \times$ $\left.\mathrm{CH}_{3}\right), 1.27(1 \mathrm{H}$, brs, $\mathrm{OH}), 1.75\left(4 \mathrm{H}\right.$, brs, $\left.2 \times \mathrm{CH}_{2}\right), 1.98\left(2 \mathrm{H}\right.$, brs, $\left.\mathrm{NCH}_{2}\right)$, $2.58-2.65\left(6 \mathrm{H}, \mathrm{m}, 3 \times \mathrm{NCH}_{2}\right), 2.84\left(2 \mathrm{H}, \mathrm{t}, J=6.0 \mathrm{~Hz}, \mathrm{NCH}_{2}\right), 3.78(1 \mathrm{H}$, brs, OCH $), 3.98\left(2 \mathrm{H}, \mathrm{t}, J=6.0 \mathrm{~Hz}, \mathrm{OCH}_{2}\right), 5.08\left(1 \mathrm{H}, \mathrm{m}, \mathrm{Ar}_{2} \mathrm{CHN}\right)$, 6.78-6.81 (2H, m, 2× Ar-H), 7.14-7.46 (6H, m, 6× Ar-H), 7.65-7.80 (2H, m, Ar-H), 13.58 (1H, brs, OH). ${ }^{13} \mathrm{C}$ NMR $(75.5 \mathrm{MHz}$, $\left.\mathrm{CDCl}_{3}\right): 12.05\left(2 \times \mathrm{CH}_{3}\right), 35.08\left(2 \times \mathrm{CH}_{2}\right), 48.12\left(2 \times \mathrm{NCH}_{2}\right), 52.05$ $\left(\mathrm{NCH}_{2}\right), 66.68\left(\mathrm{OCH}_{2}\right), 71.22\left(\mathrm{Ar}_{2} \mathrm{CHN}\right), 115.18,116.65,120.29,121.47$, 121.82, 122.80, 126.79, 127.23, 129.11, 129.27, 129.74, 130.57, 132.08, 132.64, 155.66 and 158.89 (Ar). HRMS $\mathrm{m} / \mathrm{z}$ calculated for $\mathrm{C}_{28} \mathrm{H}_{36} \mathrm{~N}_{2} \mathrm{O}_{3}[\mathrm{M}+\mathrm{H}]^{+} 449.2726$, observed $[\mathrm{M}+\mathrm{H}]^{+} 449.2773$.

5.1.3.3. 1-[[4-(2-(Diisopropylamino)ethoxy)phenyl](2-hydroxynaphthalen-1-yl) methyl]piperidin-4-ol (2c). Light brown solid, yield 44\%, m.p. $81-83{ }^{\circ} \mathrm{C}$. UV(EtOH) $\lambda_{\max }: 232,280$ and $334 \mathrm{~nm}$. IR (KBr): $3422,2965,2870,1609,1511,1466,1362,1241,1178,1052,951$, and $829 \mathrm{~cm}^{-1}$. ${ }^{\mathrm{H}} \mathrm{NMR}\left(300 \mathrm{MHz}, \mathrm{CDCl}_{3}\right): 1.04(12 \mathrm{H}, \mathrm{d}, J=6.3 \mathrm{~Hz}, 4 \times$
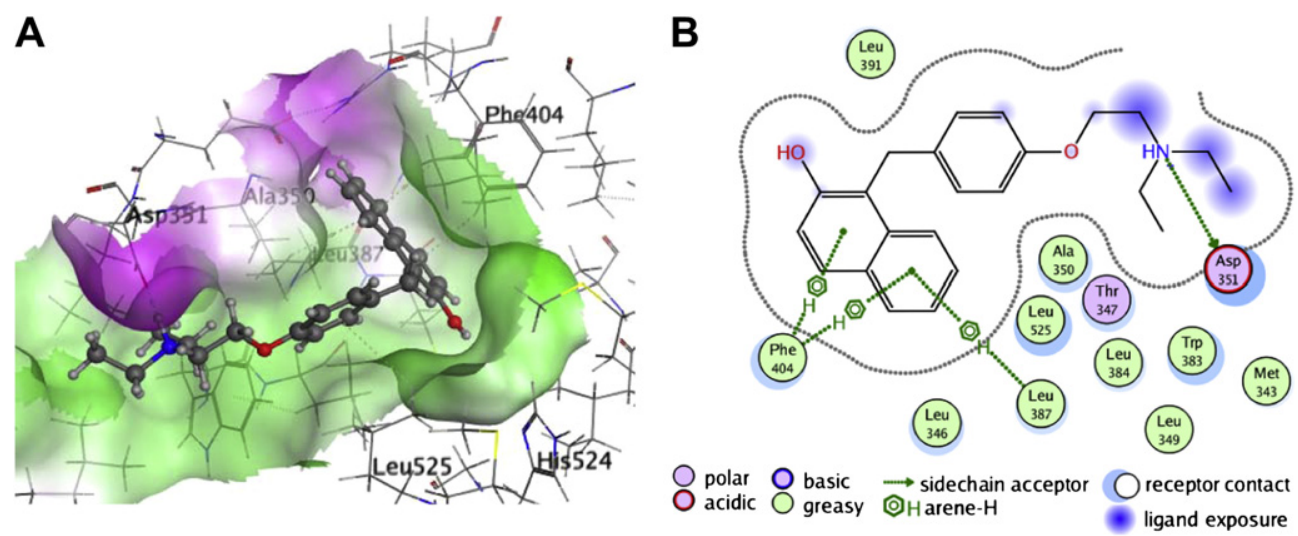

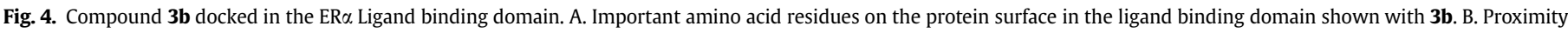
countor of $\mathbf{3 b}$ indicating important interactions with amino acid residues in the ER $\alpha$ Ligand binding domain. 


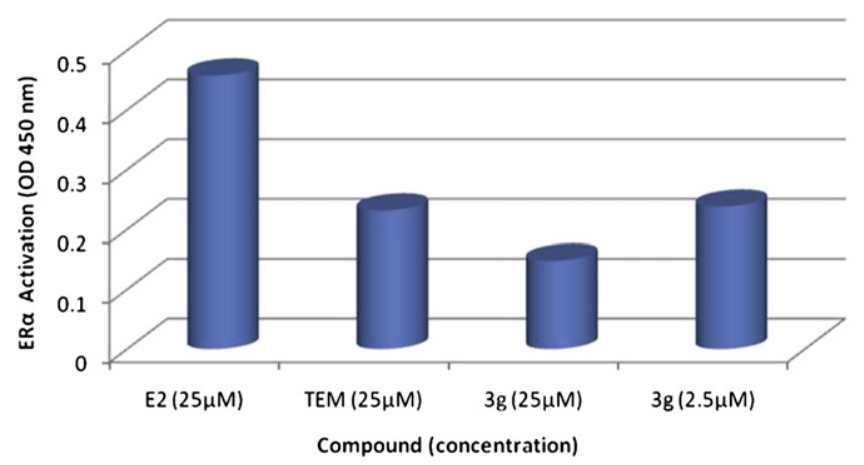

Fig. 5. Human ER $\alpha$ binding affinity tested using ActiveMotif's NR Peptide ER $\alpha$ Colorimetric ELISA kit following manufacturer's instructions. E2 $(25 \mu \mathrm{M})$ : positive (agonist) control treated with $25 \mu \mathrm{M}$ estradiol; TEM $(25 \mu \mathrm{M})$ : negative (antagonist) control treated with $25 \mu \mathrm{M}$ tamoxifen. The most potent compound from series $\mathbf{3}$, i.e. $\mathbf{3 g}$, was assayed at $25 \mu \mathrm{M}$ and $2.5 \mu \mathrm{M}$ concentration.

$\left.\mathrm{CH}_{3}\right), 1.29(1 \mathrm{H}$, brs, $\mathrm{OH}), 1.63-1.76\left(4 \mathrm{H}, \mathrm{m}, 2 \times \mathrm{CH}_{2}\right), 1.97(2 \mathrm{H}$, brs, $\left.\mathrm{NCH}_{2}\right), 2.70-2.80\left(4 \mathrm{H}, \mathrm{m}, \mathrm{NCH}_{2}\right), 3.01-3.05(2 \mathrm{H}, \mathrm{m}, 2 \times \mathrm{NCH})$, $3.81-3.86\left(3 \mathrm{H}, \mathrm{m}, \mathrm{OCH}_{2} \& \mathrm{OCH}\right), 5.08\left(1 \mathrm{H}, \mathrm{brs}, \mathrm{Ar}_{2} \mathrm{CHN}\right), 6.79-6.82$ $(2 \mathrm{H}, \mathrm{m}, 2 \times \mathrm{Ar}-\mathrm{H}), 7.16-7.28(2 \mathrm{H}, \mathrm{m}, 2 \times \mathrm{Ar}-\mathrm{H}), 7.35-7.66(3 \mathrm{H}, \mathrm{m}$, $3 \times \mathrm{Ar}-\mathrm{H}), 7.70-7.84(3 \mathrm{H}, \mathrm{m}, 3 \times \mathrm{Ar}-\mathrm{H}), 13.58(1 \mathrm{H}, \mathrm{brs}, \mathrm{OH}) .{ }^{13} \mathrm{C}$ NMR Data (75.5 MHz, $\left.\mathrm{CDCl}_{3}\right): 20.77\left(4 \times \mathrm{CH}_{3}\right), 29.72\left(\mathrm{CH}_{2}\right), 35.25$ $\left(\mathrm{CH}_{2}\right), 44.43(2 \times \mathrm{NCH}), 49.70\left(\mathrm{NCH}_{2}\right), 69.12\left(\mathrm{OCH}_{2}\right) 70.85\left(\mathrm{Ar}_{2} \mathrm{CHN}\right)$, $114.67,116.25,119.89,121.08,122.40,126.40,128.71,129.34,131.44$, 132.24, 155.24 and $158.64(\mathrm{Ar})$. HRMS $\mathrm{m} / \mathrm{z}$ calculated for $\mathrm{C}_{30} \mathrm{H}_{40} \mathrm{~N}_{2} \mathrm{O}_{3}[\mathrm{M}+\mathrm{H}]^{+}$477.3039, observed $[\mathrm{M}+\mathrm{H}]^{+} 477.3116$.

5.1.3.4. 1-[(2-Hydroxynaphthalen-1-yl)[4-[2-(pyrrolidin-1-yl)ethoxy]phenyl]methyl] piperidin-4-ol (2d). Light brown solid, yield 39\%, m.p. $102-104{ }^{\circ} \mathrm{C}$. UV(EtOH) $\lambda_{\max }: 232,279$ and $334 \mathrm{~nm}$. IR (KBr): $3404,3058,2927,2836,1608,1503,1436,1354,1239,1175,1139$, 1062 , and $816 \mathrm{~cm}^{-1} .{ }^{1} \mathrm{H}$ NMR (300 MHz, $\left.\mathrm{CDCl}_{3}\right): 1.45\left(2 \mathrm{H}\right.$, brs, $\left.\mathrm{CH}_{2}\right)$, $1.69\left(6 \mathrm{H}\right.$, brs, $\left.3 \times \mathrm{CH}_{2}\right), 1.81\left(2 \mathrm{H}\right.$, brs, $\left.\mathrm{NCH}_{2}\right), 2.31(1 \mathrm{H}$, brs, $\mathrm{OH}), 2.65$ $\left(6 \mathrm{H}\right.$, brs, $\left.3 \times \mathrm{CH}_{2}\right), 2.89\left(2 \mathrm{H}\right.$, brs, $\left.\mathrm{NCH}_{2}\right), 3.85(1 \mathrm{H}$, brs, $\mathrm{OCH}), 4.07$ $\left(2 \mathrm{H}\right.$, brs, $\left.\mathrm{OCH}_{2}\right), 5.09\left(1 \mathrm{H}\right.$, brs, $\left.\mathrm{Ar}_{2} \mathrm{CHN}\right), 6.81-6.84(2 \mathrm{H}, \mathrm{m}, 2 \times$ $\mathrm{Ar}-\mathrm{H}), 7.15-7.48(5 \mathrm{H}, \mathrm{m}, 5 \times \mathrm{Ar}-\mathrm{H}), 7.66-7.81(3 \mathrm{H}, \mathrm{m}, 3 \times \mathrm{Ar}-\mathrm{H})$. ${ }^{13} \mathrm{C}$ NMR Data (75.5 MHz, $\left.\mathrm{CDCl}_{3}\right): 23.34\left(2 \times \mathrm{CH}_{2}\right), 29.82\left(2 \times \mathrm{CH}_{2}\right)$, $54.58\left(2 \times \mathrm{NCH}_{2}\right), 55.15\left(\mathrm{NCH}_{2}\right), 66.51\left(\mathrm{OCH}_{2}\right), 71.29\left(\mathrm{Ar}_{2} \mathrm{CHN}\right)$, $114.39,118.95,119.04,122.52,123.35,126.40,128.39,129.10,129.40$, $131.40,132.57,133.22,152.12$ and 157.19. HRMS $m / z$ calculated for $\mathrm{C}_{28} \mathrm{H}_{34} \mathrm{~N}_{2} \mathrm{O}_{3}[\mathrm{M}+\mathrm{H}]^{+}$447.2569, observed $[\mathrm{M}+\mathrm{H}]^{+} 447.2773$.

5.1.3.5. 1-[(2-Hydroxynaphthalen-1-yl)[4-[2-(piperidin-1-yl)ethoxy] phenyl]methyl] piperidin-4-ol (2e). Off white solid, yield 42\%, m.p. 83-85 ${ }^{\circ} \mathrm{C}$. UV(EtOH) $\lambda_{\max }: 232,280$ and $334 \mathrm{~nm}$. IR (KBr): 3417, 2933, 2852, 1609, 1511, 1453, 1367, 1304, 1268, 1239, 1179, 1052, 946 and $829 \mathrm{~cm}^{-1}$. ${ }^{1} \mathrm{H}$ NMR $\left(\mathrm{CDCl}_{3}, 300 \mathrm{MHz}\right): 0.88\left(2 \mathrm{H}, \mathrm{brs}, \mathrm{CH}_{2}\right), 1.28$ $\left(2 \mathrm{H}\right.$, brs, $\left.\mathrm{CH}_{2}\right), 1.43-1.59\left(6 \mathrm{H}, \mathrm{m}, 3 \times \mathrm{CH}_{2}\right), 1.74(\mathrm{IH}, \mathrm{brs}, \mathrm{OH}), 1.98$ $\left(2 \mathrm{H}\right.$, brs, $\left.\mathrm{NCH}_{2}\right), 2.47\left(6 \mathrm{H}\right.$, brs, $\left.3 \times \mathrm{NCH}_{2}\right), 2.72\left(2 \mathrm{H}\right.$, brs, $\left.\mathrm{NCH}_{2}\right), 4.03$ $\left(3 \mathrm{H}\right.$, brs, $\left.\mathrm{OCH}_{2} \& \mathrm{OCH}\right), 5.07\left(1 \mathrm{H}\right.$, brs, $\left.\mathrm{Ar}_{2} \mathrm{CHN}\right), 6.80$ (2H, brs, $2 \times$ $\mathrm{Ar}-\mathrm{H}), 7.15-7.37(6 \mathrm{H}, \mathrm{m}, 6 \times \mathrm{Ar}-\mathrm{H}), 7.45-7.67(2 \mathrm{H}, \mathrm{m}, 2 \times \mathrm{Ar}-\mathrm{H})$. ${ }^{13} \mathrm{C}$ NMR (75.5 MHz, $\left.\mathrm{CDCl}_{3}\right): 24.45\left(\mathrm{CH}_{2}\right), 26.15\left(4 \times \mathrm{CH}_{2}\right), 55.31$ $\left(2 \times \mathrm{NCH}_{2}\right), 58.16\left(\mathrm{NCH}_{2}\right), 66.09\left(\mathrm{OCH}_{2}\right), 71.21\left(\mathrm{Ar}_{2} \mathrm{CHN}\right), 115.06$, 116.64, 120.29, 121.45, 122.79, 126.78, 129.11, 129.26, 129.74, 130.59, $132.14,132.63,155.64$ and 158.83. HRMS $m / z$ calculated for $\mathrm{C}_{29} \mathrm{H}_{36} \mathrm{~N}_{2} \mathrm{O}_{3}[\mathrm{M}+\mathrm{H}]^{+}$461.2726, observed $[\mathrm{M}+\mathrm{H}]^{+} 461.2785$.

5.1.3.6. 1-[(2-Hydroxynaphthalen-1-yl)[4-(2-morpholinoethoxy)phenyl]methyl piperidin-4-ol (2f). Light yellow solid, yield 47\%, m.p. 97-99 ${ }^{\circ} \mathrm{C}$. $\mathrm{UV}(\mathrm{EtOH}) \lambda_{\max }: 232,280$ and $334 \mathrm{~nm}$. IR (KBr): 3425, 2930, 2854, $1608,1511,1453,1268,1241,1179,1116,1052,946$ and $832 \mathrm{~cm}^{-1} .{ }^{1} \mathrm{H}$ NMR $\left(\mathrm{CDCl}_{3}, 300 \mathrm{MHz}\right)$ : $1.64-1.76\left(4 \mathrm{H}, \mathrm{m}, 2 \times \mathrm{CH}_{2}\right), 1.98(1 \mathrm{H}$, brs, $\mathrm{OH})$,
2.55 ( $8 \mathrm{H}$, brs, $4 \times \mathrm{NCH}_{2}$ ), $2.76\left(2 \mathrm{H}\right.$, brs, $\left.\mathrm{NCH}_{2}\right), 3.71\left(4 \mathrm{H}, \mathrm{brs}, 2 \times \mathrm{OCH}_{2}\right)$, 4.04 (3H, brs, $\left.\mathrm{OCH}_{2} \& \mathrm{OCH}\right), 5.08$ ( $\left.\mathrm{IH}, \mathrm{s}, \mathrm{Ar}_{2} \mathrm{CHN}\right), 6.79-6.82(2 \mathrm{H}, \mathrm{m}, 2 \times$ $\mathrm{Ar}-\mathrm{H}), 7.14-7.45(6 \mathrm{H}, \mathrm{m}, 6 \times \mathrm{Ar}-\mathrm{H}), 7.66-7.82(2 \mathrm{H}, \mathrm{m}, 2 \times \mathrm{Ar}-\mathrm{H}) .{ }^{13} \mathrm{C}$ $\mathrm{NMR}\left(75.5 \mathrm{MHz}, \mathrm{CDCl}_{3}\right): 26.49\left(2 \times \mathrm{CH}_{2}\right) 54.41\left(2 \times \mathrm{NCH}_{2}\right), 57.94\left(\mathrm{NCH}_{2}\right)$, $66.07\left(\mathrm{OCH}_{2}\right), 67.28\left(2 \times \mathrm{OCH}_{2}\right), 71.19\left(\mathrm{Ar}_{2} \mathrm{CHN}\right), 115.22,116.61,120.29$, $121.42,122.80,126.78,129.28,129.76,130.51,132.29,132.61,155.64$ and 158.77. HRMS $m / z$ calculated for $\mathrm{C}_{28} \mathrm{H}_{34} \mathrm{~N}_{2} \mathrm{O}_{4}[\mathrm{M}+\mathrm{H}]^{+} 463.2519$, observed $[\mathrm{M}+\mathrm{H}]^{+} 463.2553$.

5.1.4. General procedure for the synthesis of 4-[2-(4-methylpiperidin1-yl)ethoxy]benzaldehyde (4g) and 4-(2-(azepan-1-yl)ethoxy)benzal dehyde (4h)

In a dried single-neck round bottom flask, appropriate amine (4-methylpiperidine or hexamethyleneimine, $21 \mathrm{mmol}$ ), potassium carbonate $(65 \mathrm{mmol})$ and dry acetone $(80 \mathrm{ml})$ were taken and the contents refluxed for $2 \mathrm{~h}$. The reaction mixture was brought to room temperature and catalytic amount of potassium iodide was added, followed by the gradual addition of 4-(2-bromoethoxy) benzaldehyde [26] (21 mmol) dissolved in dry acetone $(50 \mathrm{ml})$ through a pressure-equalizing addition funnel and the reaction mixture was allowed to reflux again. The contents were regularly monitored for reaction progress by TLC using 10\% methanol/ dichloromethane as the solvent system. The reaction was generally completed in $10-13 \mathrm{~h}$. At this point, the reaction mixture was filtered under suction and the solid inorganic salts were washed with acetone $(3 \times 60 \mathrm{ml})$. The solvent was evaporated under reduced pressure and the residue was purified by column chromatography over silica gel (6-8\% methanol in dichloromethane, $\mathrm{v} / \mathrm{v}$ as eluent) to afford pure aldehydes $\mathbf{4 g}$ and $\mathbf{4 h}$ as colorless oils in 62 and $63 \%$ yields respectively.

5.1.4.1. 4-[2-(4-Methylpiperidin-1-yl)ethoxy]benzaldehyde (4g). Colorless oil, yield $62 \%$. UV(EtOH) $\lambda_{\max }$ : 217 and $273 \mathrm{~nm}$. IR (nujol): 2923, 2947, 2719, 2745, 1695, 1602, 1509, 1260, 1160, 1025 and $832 \mathrm{~cm}^{-1} .{ }^{1} \mathrm{H} \mathrm{NMR}\left(\mathrm{CDCl}_{3}, 300 \mathrm{MHz}\right): \delta 0.94(3 \mathrm{H}, \mathrm{d}, J=6.0 \mathrm{~Hz}$, $\left.\mathrm{CH}_{3}\right), 1.30-1.37\left(3 \mathrm{H}, \mathrm{m}, \mathrm{CH}_{2} \& \mathrm{CH}\right), 1.62-1.66\left(2 \mathrm{H}, \mathrm{m}, \mathrm{CH}_{2}\right)$, $2.09-2.16\left(2 \mathrm{H}, \mathrm{m}, \mathrm{NCH}_{2}\right), 2.83\left(2 \mathrm{H}, \mathrm{t}, J=5.7 \mathrm{~Hz}, \mathrm{NCH}_{2}\right), 2.96-3.00$ $\left(2 \mathrm{H}, \mathrm{m}, \mathrm{NCH}_{2}\right), 4.20\left(2 \mathrm{H}, \mathrm{t}, J=5.7 \mathrm{~Hz}, \mathrm{OCH}_{2}\right), 7.01(2 \mathrm{H}, \mathrm{d}$, $J=8.4 \mathrm{~Hz}, 2 \times \mathrm{Ar}-\mathrm{H}), 7.83(2 \mathrm{H}, \mathrm{d}, J=8.7 \mathrm{~Hz}, 2 \times \mathrm{Ar}-\mathrm{H}), 9.87$ (CHO). ${ }^{13} \mathrm{C}$ NMR $\left(75.5 \mathrm{MHz}, \mathrm{CDCl}_{3}\right): \delta 21.79\left(\mathrm{CH}_{3}\right), 30.45(\mathrm{CH})$, $34.32\left(2 \times \mathrm{CH}_{2}\right), 54.49\left(2 \times \mathrm{NCH}_{2}\right), 57.24\left(\mathrm{NCH}_{2}\right), 66.42\left(\mathrm{OCH}_{2}\right)$, 114.87 (C-2 \& C-6), 130.29 (C-1), 131.92 (C-3 \& C-5), 163.82 (C-4), $190.70(\mathrm{C}=\mathrm{O})$. HRMS $m / z$ calculated for $\mathrm{C}_{15} \mathrm{H}_{21} \mathrm{NO}_{2}[\mathrm{M}+\mathrm{H}]^{+}$ 248.1645, observed [M $+\mathrm{H}]^{+} 248.1646$.

5.1.4.2. 4-[2-(Azepan-1-yl)ethoxy]benzaldehyde (4h). Colorless oil, yield 63\%. UV (EtOH) $\lambda_{\text {max }}$ : 217 and $276 \mathrm{~nm}$. IR (nujol): 2924, 2851, 1694, 1600, 1509, 1312, 1258, 1159, 1022 and $832 \mathrm{~cm}^{-1} .{ }^{1} \mathrm{H}$ NMR $\left(300 \mathrm{MHz}, \mathrm{CDCl}_{3}\right): \delta 1.64-1.71\left(8 \mathrm{H}, \mathrm{m}, 4 \times \mathrm{CH}_{2}\right), 2.86(4 \mathrm{H}$, brs, $2 \times$ $\left.\mathrm{NCH}_{2}\right), 3.05\left(2 \mathrm{H}, \mathrm{t}, J=5.7 \mathrm{~Hz}, \mathrm{NCH}_{2}\right), 4.21\left(2 \mathrm{H}, \mathrm{t}, J=6.0 \mathrm{~Hz}, \mathrm{OCH}_{2}\right)$, $7.03(2 \mathrm{H}, \mathrm{d}, J=8.4 \mathrm{~Hz}, 2 \times \mathrm{Ar}-\mathrm{H}), 7.85(2 \mathrm{H}, \mathrm{d}, J=8.4 \mathrm{~Hz}, 2 \times \mathrm{Ar}-\mathrm{H})$, $9.89(\mathrm{CHO}) .{ }^{13} \mathrm{C} \mathrm{NMR}\left(75.5 \mathrm{MHz}, \mathrm{CDCl}_{3}\right): \delta 27.02\left(2 \times \mathrm{CH}_{2}\right), 27.87(2 \times$ $\left.\mathrm{CH}_{2}\right), 55.82\left(2 \times \mathrm{NCH}_{2}\right), 57.95\left(\mathrm{NCH}_{2}\right), 66.89\left(\mathrm{OCH}_{2}\right), 114.86(\mathrm{C}-2$ \& C-6), 129.90 (C-1), 131.92 (C-3 \& C-5), 163.96 (C-4), 190.72 (C=0). HRMS $m / z$ calculated for $\mathrm{C}_{15} \mathrm{H}_{21} \mathrm{NO}_{2}[\mathrm{M}+\mathrm{H}]^{+} 248.1645$, observed $[\mathrm{M}+\mathrm{H}]^{+} 248.1649$.

\subsubsection{General procedure for the synthesis of 4-(dialkylaminoethoxy) benzyl alcohols $\mathbf{5 a}-\boldsymbol{h}$}

In a round bottom flask, a solution of the compound $\mathbf{4 a - h}$ $(4.5 \mathrm{mmol})$ in methanol $(25 \mathrm{ml})$ was taken and sodium borohydride (3.1 mmol) was added in small lots at room temperature. The reaction was monitored by TLC using $10 \%$ methanol/dichloromethane as the solvent system. The reaction was completed in 3-5 h. After completion of reaction, the methanol was removed under reduced 
pressure. Water $(15 \mathrm{ml})$ was added and the reaction mixture was extracted with ethyl acetate $(3 \times 50 \mathrm{ml})$. The organic layer was washed with water $(3 \times 50 \mathrm{ml})$ and dried over anhydrous sodium sulfate. The solvent was removed under reduced pressure and dried under high vacuum to afford the desired alcohols $\mathbf{5 a} \mathbf{a}-\mathbf{h}$ as colorless oils in 76-95\% yields. The structures of the compounds $\mathbf{5 a}-\mathbf{h}$ were unambiguously established by comparing their spectral data with reported values [14]. Compound $\mathbf{5 g}$ was found to be new to chemical literature; its spectral data is reported below.

5.1.5.1. [4-[2-(4-Methylpiperidin-1-yl)ethoxy]phenyl]methanol (5g). Colorless oil, yield $88 \%$. UV (EtOH) $\lambda_{\max }: 225$ and $272 \mathrm{~nm}$. IR (nujol): 3361, 2932, 2868, 1611, 1511, 1455, 1321, 1298, 1244, 1172, 1053, 1009 and $821 \mathrm{~cm}^{-1}$. ${ }^{1} \mathrm{H}$ NMR $\left(300 \mathrm{MHz}, \mathrm{CDCl}_{3}\right): \delta 0.95(3 \mathrm{H}, \mathrm{d}, J=5.4 \mathrm{~Hz}$, $\left.\mathrm{CH}_{3}\right), 1.28-1.36\left(3 \mathrm{H}, \mathrm{m}, \mathrm{CH}_{2} \& \mathrm{CH}\right), 1.63-1.67\left(2 \mathrm{H}, \mathrm{m}, \mathrm{CH}_{2}\right)$, $2.08-2.16\left(2 \mathrm{H}, \mathrm{m}, \mathrm{NCH}_{2}\right), 2.79\left(2 \mathrm{H}, \mathrm{t}, J=6.0 \mathrm{~Hz}, \mathrm{NCH}_{2}\right), 2.96-3.00$ $\left(2 \mathrm{H}, \mathrm{m}, \mathrm{NCH}_{2}\right), 4.10\left(2 \mathrm{H}, \mathrm{t}, J=5.7 \mathrm{~Hz}, \mathrm{OCH}_{2}\right), 4.62\left(2 \mathrm{H}, \mathrm{s}, \mathrm{CH}_{2} \mathrm{OH}\right)$, $6.89(2 \mathrm{H}, \mathrm{d}, J=7.8 \mathrm{~Hz}, 2 \times \mathrm{Ar}-\mathrm{H}), 7.30(2 \mathrm{H}, \mathrm{d}, J=7.5 \mathrm{~Hz}, 2 \times \mathrm{Ar}-\mathrm{H})$. ${ }^{13} \mathrm{C}$ NMR $\left(75.5 \mathrm{MHz}, \mathrm{CDCl}_{3}\right): \delta 21.81\left(\mathrm{CH}_{3}\right), 30.51(\mathrm{CH}), 34.10(2 \times$ $\left.\mathrm{CH}_{2}\right), 54.46\left(2 \times \mathrm{NCH}_{2}\right), 57.48\left(\mathrm{NCH}_{2}\right), 64.88\left(\mathrm{CH}_{2} \mathrm{OH}\right), 66.00\left(\mathrm{OCH}_{2}\right)$, 114.65 (C-2 \& C-6), 128.55 (C-3 \& C-5), 133.56 (C-1), 158.36 (C-4). HRMS $m / z$ calculated for $\mathrm{C}_{15} \mathrm{H}_{23} \mathrm{NO}_{2}[\mathrm{M}+\mathrm{H}]^{+} 250.1802$, observed $[\mathrm{M}+\mathrm{H}]^{+} 250.1802$.

\subsubsection{General procedure for the synthesis of compounds $\mathbf{3 a}-\boldsymbol{h}$}

To a solution of $\mathbf{4 a}-\mathbf{h}(12.7 \mathrm{mmol})$ and 2-naphthol $(12.7 \mathrm{mmol})$ in dry 1,4-dioxane $(60 \mathrm{ml})$, boron trifluoride etherate $(38 \mathrm{mmol})$ was added slowly at $0{ }^{\circ} \mathrm{C}$. After the addition of boron trifluoride etherate, the reaction mixture was stirred at room temperature until TLC showed completion of reaction. The reaction was generally complete in $3-5 \mathrm{~h}$. After the completion of reaction, ethyl acetate was added and the reaction mixture was washed with saturated solution of sodium bicarbonate $(3 \times 100 \mathrm{ml})$, followed by water $(3 \times 100 \mathrm{ml})$ and with brine $(3 \times 100 \mathrm{ml})$. The organic layer was dried over anhydrous sodium sulfate. It was then evaporated under reduced pressure to obtain the crude product, which was purified by column chromatography over silica gel using methanoldichloromethane (8-10\%) as eluent to afford pure compounds $\mathbf{3 a}-\mathbf{h}$ in $45-70 \%$ yields.

5.1.6.1. 1-[4-[2-(Dimethylamino)ethoxy]benzyl]naphthalen-2-ol (3a). Off white solid, yield $45 \%$, m.p. $122-125^{\circ} \mathrm{C}$. UV (EtOH) $\lambda_{\max }: 229,280$ and $334 \mathrm{~nm}$. IR (KBr): 3370, 3060, 2938, 2825, 1593, 1507, 1465, 1369, $1244,1176,1078,1045$ and $806 \mathrm{~cm}^{-1} .{ }^{1} \mathrm{H} \mathrm{NMR}\left(300 \mathrm{MHz}, \mathrm{CDCl}_{3}\right) \delta 2.43$ $\left(6 \mathrm{H}, \mathrm{s}, 2 \times \mathrm{NCH}_{3}\right), 2.83\left(2 \mathrm{H}, \mathrm{t}, J=5.4 \mathrm{~Hz},-\mathrm{NCH}_{2}\right), 4.01(2 \mathrm{H}, \mathrm{t}, J=5.4 \mathrm{~Hz}$, $\left.\mathrm{OCH}_{2}\right), 4.37\left(2 \mathrm{H}, \mathrm{s}, \mathrm{ArCH}_{2} \mathrm{Ar}\right), 6.66(2 \mathrm{H}, \mathrm{d}, J=8.1 \mathrm{~Hz}, 2 \times \mathrm{Ar}-\mathrm{H}), 7.08$ $(2 \mathrm{H}, \mathrm{d}, J=8.1 \mathrm{~Hz}, 2 \times \mathrm{Ar}-\mathrm{H}), 7.18(1 \mathrm{H}, \mathrm{d}, J=9.0 \mathrm{~Hz}, \mathrm{Ar}-\mathrm{H}), 7.30(1 \mathrm{H}, \mathrm{t}$, $J=6.9 \mathrm{~Hz}, \mathrm{Ar}-\mathrm{H}), 7.42(1 \mathrm{H}, \mathrm{t}, J=7.8 \mathrm{~Hz}, \mathrm{Ar}-\mathrm{H}), 7.66(1 \mathrm{H}, \mathrm{d}, J=8.7 \mathrm{~Hz}$, $\mathrm{Ar}-\mathrm{H}), 7.77(1 \mathrm{H}, \mathrm{d}, J=8.1 \mathrm{~Hz}, \mathrm{Ar}-\mathrm{H}), 7.88(1 \mathrm{H}, \mathrm{d}, J=8.7 \mathrm{~Hz}, \mathrm{Ar}-\mathrm{H}) .{ }^{13} \mathrm{C}$ NMR (75.5 MHz, CDCl 3 ): $\delta 29.77\left(\mathrm{ArCH}_{2} \mathrm{Ar}\right), 45.22\left(2 \times \mathrm{NCH}_{3}\right), 57.89$ $\left(\mathrm{NCH}_{2}\right), 64.81\left(\mathrm{OCH}_{2}\right), 114.42(\mathrm{Ar}), 118.26(\mathrm{Ar}), 118.72(\mathrm{Ar}), 122.64(\mathrm{Ar})$, 123.29 (Ar), 126.31 (Ar), 128.08 (Ar), 128.46 (Ar), 129.04 (Ar), 129.22 (Ar), 133.39 (Ar), 133.78 (Ar), 152.16 (Ar), 156.50 (Ar). HRMS m/z calculated for $\mathrm{C}_{21} \mathrm{H}_{23} \mathrm{NO}_{2}[\mathrm{M}+\mathrm{H}]^{+} 322.1802$, observed $[\mathrm{M}+\mathrm{H}]^{+}$ 322.1790

5.1.6.2. 1-[4-[2-(Diethylamino)ethoxy]benzyl]naphthalen-2-ol(3b). Off white solid, yield 65\%, m.p. $105-107^{\circ} \mathrm{C}$. UV (EtOH) $\lambda_{\max }: 229,279$ and 334 nm. IR (KBr): 3411, 3059, 2973, 2935, 2857, 1582, 1507, 1443, 1354, 1241, 1176, 1036, 994 and $804 \mathrm{~cm}^{-1}$. ${ }^{1} \mathrm{H}$ NMR (300 MHz, $\left.\mathrm{CDCl}_{3}\right): \delta 1.18$ $\left(6 \mathrm{H}, \mathrm{t}, J=7.2 \mathrm{~Hz}, 2 \times \mathrm{CH}_{3}\right), 2.76\left(4 \mathrm{H}, \mathrm{q}, J=7.2 \mathrm{~Hz}, 2 \times \mathrm{NCH}_{2}\right), 2.93(2 \mathrm{H}, \mathrm{t}$, $\left.J=6.0 \mathrm{~Hz},-\mathrm{NCH}_{2}\right), 4.02\left(2 \mathrm{H}, \mathrm{t}, J=6.0 \mathrm{~Hz}, \mathrm{OCH}_{2}\right), 4.37(2 \mathrm{H}, \mathrm{s}$, $\left.\mathrm{ArCH}_{2} \mathrm{Ar}\right), 6.68(2 \mathrm{H}, \mathrm{d}, J=8.4 \mathrm{~Hz}, 2 \times \mathrm{Ar}-\mathrm{H}), 7.09(2 \mathrm{H}, \mathrm{d}, J=8.4 \mathrm{~Hz}$, $2 \times \mathrm{Ar}-\mathrm{H}), 7.17(1 \mathrm{H}, \mathrm{d}, J=8.7 \mathrm{~Hz}, \mathrm{Ar}-\mathrm{H}), 7.32(1 \mathrm{H}, \mathrm{t}, J=7.8 \mathrm{~Hz}, \mathrm{Ar}-\mathrm{H})$, $7.43(1 \mathrm{H}, \mathrm{t}, J=6.9 \mathrm{~Hz}, \mathrm{Ar}-\mathrm{H}), 7.65(2 \mathrm{H}, \mathrm{d}, J=9.0 \mathrm{~Hz}, \mathrm{Ar}-\mathrm{H}), 7.76(1 \mathrm{H}, \mathrm{d}$,
$J=8.1 \mathrm{~Hz}, \mathrm{Ar}-\mathrm{H}), 7.89(1 \mathrm{H}, \mathrm{d}, J=8.4 \mathrm{~Hz}, \mathrm{Ar}-\mathrm{H}) .{ }^{13} \mathrm{C}$ NMR $(75.5 \mathrm{MHz}$, $\left.\mathrm{CDCl}_{3}\right): \delta 10.70\left(2 \times \mathrm{CH}_{3}\right), 29.82\left(\mathrm{ArCH}_{2} \mathrm{Ar}\right), 47.32\left(2 \times \mathrm{NCH}_{2}\right), 51.57$ $\left(\mathrm{NCH}_{2}\right), 65.39\left(\mathrm{OCH}_{2}\right), 114.41(\mathrm{Ar}), 118.33(\mathrm{Ar}), 118.86(\mathrm{Ar}), 122.72(\mathrm{Ar})$, 123.32 (Ar), 126.34 (Ar), 128.10 (Ar), 128.47 (Ar), 129.14 (Ar), 129.21 (Ar), $133.10(\mathrm{Ar}), 133.78(\mathrm{Ar}), 151.97(\mathrm{Ar}), 156.67(\mathrm{Ar}) . \mathrm{HRMS} \mathrm{m} / \mathrm{z}$ calculated for $\mathrm{C}_{23} \mathrm{H}_{27} \mathrm{NO}_{2}[\mathrm{M}+\mathrm{H}]^{+} 350.2115$, observed $[\mathrm{M}+\mathrm{H}]^{+}$ 350.2101 .

5.1.6.3. 1-[4-[2-(Diisopropylamino)ethoxy]benzyl]naphthalen-2-ol (3c). Brown viscous oil, yield 68\%. UV (EtOH) $\lambda_{\text {max }}: 229,277$ and $334 \mathrm{~nm}$. IR (nujol): 3385, 3057, 2967, 1610, 1507, 1436, 1359, 1242, 1174, 1029, 991 and $810 \mathrm{~cm}^{-1}$. ${ }^{1} \mathrm{H}$ NMR (300 MHz, CDCl $) \delta 1.10(12 \mathrm{H}, \mathrm{d}, J=3.9 \mathrm{~Hz}$, $\left.4 \times \mathrm{CH}_{3}\right), 2.88\left(2 \mathrm{H}, \mathrm{brs},-\mathrm{NCH}_{2}\right), 3.12(2 \mathrm{H}, \mathrm{brs}, 2 \times \mathrm{NCH}), 3.92(2 \mathrm{H}, \mathrm{brs}$, $\left.\mathrm{OCH}_{2}\right), 4.40\left(2 \mathrm{H}, \mathrm{s}, \mathrm{ArCH}_{2} \mathrm{Ar}\right), 6.79(2 \mathrm{H}, \mathrm{d}, J=7.2 \mathrm{~Hz}, 2 \times \mathrm{Ar}-\mathrm{H}), 7.14(2 \mathrm{H}$, $\mathrm{d}, J=7.2 \mathrm{~Hz}, 2 \times \mathrm{Ar}-\mathrm{H}), 7.20(1 \mathrm{H}, \mathrm{d}, J=8.7 \mathrm{~Hz}, \mathrm{Ar}-\mathrm{H}), 7.36(1 \mathrm{H}, \mathrm{t}$, $J=6.6 \mathrm{~Hz}, \mathrm{Ar}-\mathrm{H}), 7.48(1 \mathrm{H}, \mathrm{t}, J=6.3 \mathrm{~Hz}, \mathrm{Ar}-\mathrm{H}), 7.71(1 \mathrm{H}, \mathrm{d}, J=8.4 \mathrm{~Hz}$, $\mathrm{Ar}-\mathrm{H}), 7.82(1 \mathrm{H}, \mathrm{d}, J=7.5 \mathrm{~Hz}, \mathrm{Ar}-\mathrm{H}), 7.95(1 \mathrm{H}, \mathrm{d}, J=8.1 \mathrm{~Hz}, \mathrm{Ar}-\mathrm{H}) .{ }^{13} \mathrm{C}$ NMR (75.0 MHz, $\left.\mathrm{CDCl}_{3}\right): \delta 20.55\left(4 \times \mathrm{CH}_{3}\right), 29.85\left(\mathrm{ArCH}_{2} \mathrm{Ar}\right), 44.67$ $(2 \times \mathrm{NCH}), 50.14\left(\mathrm{NCH}_{2}\right), 68.74\left(\mathrm{OCH}_{2}\right), 114.57(\mathrm{Ar}), 118.18(\mathrm{Ar}), 118.73$ (Ar), 123.03 (Ar), $123.37(\mathrm{Ar}), 126.53(\mathrm{Ar}), 128.30(\mathrm{Ar}), 128.53(\mathrm{Ar})$, 129.19 (Ar), 129.40 (Ar), 132.23 (Ar), 133.72 (Ar), 151.49( $\mathrm{Ar}), 157.16(\mathrm{Ar})$. HRMS $m / z$ calculated for $\mathrm{C}_{25} \mathrm{H}_{31} \mathrm{NO}_{2}[\mathrm{M}+\mathrm{H}]^{+} 378.2428$, observed $[\mathrm{M}+\mathrm{H}]^{+}$378.2417.

5.1.6.4. 1-[4-[2-(Pyrrolidin-1-yl)ethoxy]benzyl]naphthalen-2-ol (3d). Off white solid, yield 56\%, m.p. 117-119 ${ }^{\circ} \mathrm{C}$. UV (EtOH) $\lambda_{\max }: 229,278$ and $337 \mathrm{~nm}$. IR (KBr): 3436, 3054, 2930, 2874, 1626, 1511, 1438, 1357, 1242, 1174, 1057, 1000 and $812 \mathrm{~cm}^{-1}$. ${ }^{1} \mathrm{H}$ NMR (300 MHz, $\mathrm{CDCl}_{3}$ ): $\delta 1.87\left(4 \mathrm{H}\right.$, brs, $\left.2 \times \mathrm{CH}_{2}\right), 2.84\left(4 \mathrm{H}\right.$, brs, $\left.2 \times \mathrm{NCH}_{2}\right), 3.00(2 \mathrm{H}$, brs, $\left.\mathrm{NCH}_{2}\right), 4.06\left(2 \mathrm{H}, \mathrm{t}, J=4.8 \mathrm{~Hz}, \mathrm{OCH}_{2}\right), 4.35\left(2 \mathrm{H}, \mathrm{s}, \mathrm{ArCH}_{2} \mathrm{Ar}\right), 6.64(2 \mathrm{H}$, $\mathrm{d}, J=7.5 \mathrm{~Hz}, 2 \times \mathrm{Ar}-\mathrm{H}), 7.06(2 \mathrm{H}, \mathrm{d}, J=7.8 \mathrm{~Hz}, 2 \times \mathrm{Ar}-\mathrm{H}), 7.20-7.30$ $(2 \mathrm{H}, \mathrm{m}, \mathrm{Ar}-\mathrm{H}), 7.40(1 \mathrm{H}, \mathrm{t}, J=7.2 \mathrm{~Hz}, \mathrm{Ar}-\mathrm{H}), 7.64(1 \mathrm{H}, \mathrm{d}, J=8.7 \mathrm{~Hz}$, $\mathrm{Ar}-\mathrm{H}), 7.75(1 \mathrm{H}, \mathrm{d}, J=8.1 \mathrm{~Hz}, \mathrm{Ar}-\mathrm{H}), 7.86(1 \mathrm{H}, \mathrm{d}, J=8.4 \mathrm{~Hz}, \mathrm{Ar}-\mathrm{H})$. ${ }^{13} \mathrm{C}$ NMR Data (75.5 MHz, $\left.\mathrm{CDCl}_{3}\right): \delta 23.28\left(2 \times \mathrm{CH}_{2}\right), 29.76\left(\mathrm{ArCH}_{2} \mathrm{Ar}\right)$, $54.47\left(2 \times \mathrm{NCH}_{2}\right), 54.75\left(\mathrm{NCH}_{2}\right), 65.45\left(\mathrm{OCH}_{2}\right), 114.42(\mathrm{Ar}), 118.24$ (Ar), $118.67(\mathrm{Ar}), 122.63(\mathrm{Ar}), 123.28(\mathrm{Ar}), 126.30(\mathrm{Ar}), 128.08(\mathrm{Ar})$, 128.46 (Ar), 129.05 (Ar), 129.23 (Ar), 133.44 (Ar), 133.77 (Ar), 152.17 (Ar), 156.40 (Ar). HRMS $m / z$ calculated for $\mathrm{C}_{23} \mathrm{H}_{25} \mathrm{NO}_{2}[\mathrm{M}+\mathrm{H}]^{+}$ 348.1958, observed $[\mathrm{M}+\mathrm{H}]^{+}$348.1952.

5.1.6.5. 1-[4-[2-(Piperidin-1-yl)ethoxy]benzyl]naphthalen-2-ol (3e). Off white solid, yield 52\%, m.p. $171-173{ }^{\circ} \mathrm{C}$. UV (EtOH) $\lambda_{\max }$ : 227, 279 and $334 \mathrm{~nm}$. IR (KBr): 3358, 3055, 2937, 2845, 1582, 1508, 1435, 1355, 1274, 1174, 1039, 996 and $810 \mathrm{~cm}^{-1}$. ${ }^{1} \mathrm{H}$ NMR $(300 \mathrm{MHz}$, $\left.\mathrm{CDCl}_{3}\right) \delta 1.38-1.78\left(6 \mathrm{H}, \mathrm{m}, 3 \times \mathrm{CH}_{2}\right), 2.97\left(2 \mathrm{H}\right.$, brs, $\left.\mathrm{NCH}_{2}\right), 3.44(4 \mathrm{H}$, brs, $\left.2 \times \mathrm{NCH}_{2}\right), 4.24\left(2 \mathrm{H}\right.$, brs, $\left.\mathrm{OCH}_{2}\right), 4.30\left(2 \mathrm{H}, \mathrm{s}, \mathrm{ArCH}_{2} \mathrm{Ar}\right), 6.87$ $(2 \mathrm{H}, \mathrm{d}, J=7.8 \mathrm{~Hz}, 2 \times \mathrm{Ar}-\mathrm{H}), 7.19(2 \mathrm{H}, \mathrm{d}, J=8.1 \mathrm{~Hz}, 2 \times \mathrm{Ar}-\mathrm{H}), 7.27$ $(2 \mathrm{H}, \mathrm{d}, J=8.1 \mathrm{~Hz}, 2 \times \mathrm{Ar}-\mathrm{H}), 7.40(1 \mathrm{H}, \mathrm{t}, J=7.2 \mathrm{~Hz}, \mathrm{Ar}-\mathrm{H}), 7.71$ $(1 \mathrm{H}, \mathrm{d}, J=8.7 \mathrm{~Hz}, \mathrm{Ar}-\mathrm{H}), 7.79(1 \mathrm{H}, \mathrm{d}, J=8.1 \mathrm{~Hz}, \mathrm{Ar}-\mathrm{H}), 7.86(1 \mathrm{H}, \mathrm{d}$, $J=8.4 \mathrm{~Hz}, \mathrm{Ar}-\mathrm{H}), 9.63$ (IH, brs, $\left.\mathrm{OH}) .{ }^{13} \mathrm{C} \mathrm{NMR} \mathrm{(75.5} \mathrm{MHz,} \mathrm{CDCl}_{3}\right)$ : o $21.60\left(\mathrm{CH}_{2}\right), 22.91\left(2 \times \mathrm{CH}_{2}\right), 29.47\left(\mathrm{ArCH}_{2} \mathrm{Ar}\right), 53.19\left(2 \times \mathrm{NCH}_{2}\right)$, $55.36\left(\mathrm{NCH}_{2}\right), 62.54\left(\mathrm{OCH}_{2}\right), 114.97(\mathrm{Ar}), 118.63(\mathrm{Ar}), 118.69(\mathrm{Ar})$, 122.70 ( $\mathrm{Ar}), 123.47$ ( $\mathrm{Ar}), 126.55$ (Ar), 128.20 (Ar), 128.79 ( $\mathrm{Ar}$ ), 129.66 (Ar), 133.68 (Ar), 134.79 (Ar), 152.92 (Ar), 155.99 (Ar). HRMS $m / z$ calculated for $\mathrm{C}_{24} \mathrm{H}_{27} \mathrm{NO}_{2}[\mathrm{M}+\mathrm{H}]^{+} 362.2115$, observed $[\mathrm{M}+\mathrm{H}]^{+}$362.2112.

5.1.6.6. 1-[4-(2-Morpholinoethoxy)benzyl]naphthalen-2-ol (3f). Off white solid, yield 70\%, m.p. $129-132{ }^{\circ} \mathrm{C}$. UV (EtOH) $\lambda_{\max }: 227,277$ and $336 \mathrm{~nm}$. IR (KBr): 3378, 2935, 2866, 2813, 1610, 1511, 1438, 1358, 1297, 1240, 1059, 982 and $815 \mathrm{~cm}^{-1} .{ }^{1} \mathrm{H}$ NMR $(300 \mathrm{MHz}$, $\left.\mathrm{CDCl}_{3}\right): \delta 2.69\left(4 \mathrm{H}\right.$, brs, $\left.2 \times \mathrm{NCH}_{2}\right), 2.86\left(2 \mathrm{H}, \mathrm{t}, J=5.1 \mathrm{~Hz}, \mathrm{NCH}_{2}\right), 3.78$ $\left(4 \mathrm{H}\right.$, brs, $\left.2 \times \mathrm{OCH}_{2}\right), 4.07\left(2 \mathrm{H}, \mathrm{t}, J=4.8 \mathrm{~Hz}, \mathrm{OCH}_{2}\right), 4.37(2 \mathrm{H}, \mathrm{s}$, $\left.\mathrm{ArCH}_{2} \mathrm{Ar}\right), 6.69(2 \mathrm{H}, \mathrm{d}, J=8.1 \mathrm{~Hz}, 2 \times \mathrm{Ar}-\mathrm{H}), 7.08(2 \mathrm{H}, \mathrm{d}, J=8.1 \mathrm{~Hz}$, $2 \times \mathrm{Ar}-\mathrm{H}), 7.19(1 \mathrm{H}, \mathrm{d}, J=8.7 \mathrm{~Hz}, \mathrm{Ar}-\mathrm{H}), 7.82-7.34(1 \mathrm{H}, \mathrm{m}, \mathrm{Ar}-\mathrm{H})$, 
$7.42(1 \mathrm{H}, \mathrm{t}, J=7.2 \mathrm{~Hz}, \mathrm{Ar}-\mathrm{H}), 7.67(1 \mathrm{H}, \mathrm{d}, J=8.7 \mathrm{~Hz}, \mathrm{Ar}-\mathrm{H}), 7.78(1 \mathrm{H}$, d, $J=8.1 \mathrm{~Hz}, \mathrm{Ar}-\mathrm{H}), 7.88(1 \mathrm{H}, \mathrm{d}, J=8.4 \mathrm{~Hz}, \mathrm{Ar}-\mathrm{H}) .{ }^{13} \mathrm{C} \mathrm{NMR}$ (75.5 MHz, $\left.\mathrm{CDCl}_{3}\right)$ : $\delta 29.76\left(\mathrm{ArCH}_{2} \mathrm{Ar}\right), 53.69\left(2 \times \mathrm{NCH}_{2}\right), 57.46$ $\left(\mathrm{NCH}_{2}\right), 64.73\left(\mathrm{OCH}_{2}\right), 66.06\left(2 \times \mathrm{OCH}_{2}\right), 114.56(\mathrm{Ar}), 118.08(\mathrm{Ar})$, 118.53 (Ar), 122.96 ( $\mathrm{Ar}), 123.29$ ( $\mathrm{Ar}), 126.51$ (Ar), 128.29 ( $\mathrm{Ar}), 128.53$ (Ar), 129.22 (Ar), 129.29 (Ar), 133.06 (Ar), 134.71 (Ar), 151.56 (Ar), 156.54 (Ar). HRMS $m / z$ calculated for $\mathrm{C}_{23} \mathrm{H}_{25} \mathrm{NO}_{3}[\mathrm{M}+\mathrm{H}]^{+} 364.1907$, observed $[\mathrm{M}+\mathrm{H}]^{+} 364.1904$.

5.1.6.7. 1-[4-[2-(4-Methylpiperidin-1-yl)ethoxy]benzyl]naphthalen-2-ol (3g). Off white solid, yield 65\%, m.p.154-156 ${ }^{\circ} \mathrm{C}$. UV (EtOH) $\lambda_{\max }$ : 229, 279 and $334 \mathrm{~nm}$. IR (KBr): 3408, 3057, 2947, 2930, 2869, 2795, $1608,1507,1357,1267,1241,1177,1060,984$ and $814 \mathrm{~cm}^{-1} .{ }^{1} \mathrm{H}$ NMR $\left(300 \mathrm{MHz}, \mathrm{CDCl}_{3}\right): \delta 0.85\left(3 \mathrm{H}, \mathrm{d}, J=6.3 \mathrm{~Hz}, \mathrm{CH}_{3}\right), 1.03-1.16(2 \mathrm{H}, \mathrm{m}$, $\left.\mathrm{CH}_{2}\right), 1.22-1.32(1 \mathrm{H}, \mathrm{m}, \mathrm{CH}), 1.50-1.54\left(2 \mathrm{H}, \mathrm{m}, \mathrm{CH}_{2}\right), 1.90-1.97(2 \mathrm{H}$, $\left.\mathrm{m}, \mathrm{NCH}_{2}\right), 2.61\left(2 \mathrm{H}, \mathrm{t}, J=5.7 \mathrm{~Hz}, \mathrm{NCH}_{2}\right), 2.81-2.85\left(2 \mathrm{H}, \mathrm{m}, \mathrm{NCH}_{2}\right)$, $3.96\left(2 \mathrm{H}, \mathrm{t}, J=6.0 \mathrm{~Hz}, \mathrm{OCH}_{2}\right), 4.28\left(2 \mathrm{H}, \mathrm{s}, \mathrm{ArCH}_{2} \mathrm{Ar}\right), 6.76(2 \mathrm{H}, \mathrm{d}$, $J=8.4 \mathrm{~Hz}, 2 \times \mathrm{Ar}-\mathrm{H}), 7.11(2 \mathrm{H}, \mathrm{d}, J=8.4 \mathrm{~Hz}, 2 \times \mathrm{Ar}-\mathrm{H}), 7.21-7.27$ $(2 \mathrm{H}, \mathrm{m}, 2 \times \mathrm{Ar}-\mathrm{H}), 7.37(1 \mathrm{H}, \mathrm{t}, J=7.2 \mathrm{~Hz}, \mathrm{Ar}-\mathrm{H}), 7.68(1 \mathrm{H}, \mathrm{d}$, $J=9.0 \mathrm{~Hz}, \mathrm{Ar}-\mathrm{H}), 7.77(1 \mathrm{H}, \mathrm{d}, J=8.1 \mathrm{~Hz}, \mathrm{Ar}-\mathrm{H}), 7.85(1 \mathrm{H}, \mathrm{d}$, $J=8.4 \mathrm{~Hz}, \mathrm{Ar}-\mathrm{H}) .{ }^{13} \mathrm{C} \mathrm{NMR}\left(75.5 \mathrm{MHz}, \mathrm{CDCl}_{3}\right): \delta 22.28\left(\mathrm{CH}_{3}\right), 29.48$ $\left(\mathrm{ArCH} \mathrm{H}_{2} \mathrm{Ar}\right), 30.64(\mathrm{CH}), 34.45\left(2 \times \mathrm{CH}_{2}\right), 54.24\left(2 \times \mathrm{NCH}_{2}\right), 57.49$ $\left(\mathrm{NCH}_{2}\right), 66.04\left(\mathrm{OCH}_{2}\right), 114.66(\mathrm{Ar}), 118.65(\mathrm{Ar}), 118.81(\mathrm{Ar}), 122.66$ (Ar), 123.51 ( $\mathrm{Ar}), 126.53(\mathrm{Ar}), 128.11(\mathrm{Ar}), 128.75(\mathrm{Ar}), 129.51(\mathrm{Ar})$, 133.69 (Ar), 133.74 (Ar), 152.93 (Ar), 156.94 (Ar). HRMS m/z calculated for $\mathrm{C}_{25} \mathrm{H}_{29} \mathrm{NO}_{2}[\mathrm{M}+\mathrm{H}]^{+} 376.2271$, observed $[\mathrm{M}+\mathrm{H}]^{+}$ 376.2266 .

5.1.6.8. 1-[4-[2-(Azepan-1-yl)ethoxy]benzyl]naphthalen-2-ol (3h). Off white solid, yield $70 \%$, m.p. $125-128{ }^{\circ} \mathrm{C}$. UV (EtOH) $\lambda_{\max }: 229$, 276 and $336 \mathrm{~nm}$. IR (KBr): 3415, 3060, 2926, 2851,1609, 1508, 1435, $1354,1257,1242,1174,1034,994$ and $804 \mathrm{~cm}^{-1}$. ${ }^{1} \mathrm{H}$ NMR $(300 \mathrm{MHz}$, $\left.\mathrm{CDCl}_{3}\right): \delta 1.62-1.71\left(8 \mathrm{H}, \mathrm{m}, 4 \times \mathrm{CH}_{2}\right), 2.83-2.86\left(4 \mathrm{H}, \mathrm{m}, 2 \times \mathrm{NCH}_{2}\right)$, $2.98\left(2 \mathrm{H}, \mathrm{t}, J=5.4 \mathrm{~Hz}, \mathrm{NCH}_{2}\right), 4.04\left(2 \mathrm{H}, \mathrm{t}, J=5.7 \mathrm{~Hz}, \mathrm{OCH}_{2}\right), 4.38$ $\left(2 \mathrm{H}, \mathrm{s}, \mathrm{ArCH}_{2} \mathrm{Ar}\right), 6.72(2 \mathrm{H}, \mathrm{d}, J=8.4 \mathrm{~Hz}, 2 \times \mathrm{Ar}-\mathrm{H}), 7.10(2 \mathrm{H}, \mathrm{d}$, $J=8.1 \mathrm{~Hz}, 2 \times \mathrm{Ar}-\mathrm{H}), 7.20(1 \mathrm{H}, \mathrm{d}, J=8.7 \mathrm{~Hz}, \mathrm{Ar}-\mathrm{H}), 7.32(1 \mathrm{H}$, t, $J=8.1 \mathrm{~Hz}, \mathrm{Ar}-\mathrm{H}), 7.43(1 \mathrm{H}, \mathrm{t}, J=7.2 \mathrm{~Hz}, \mathrm{Ar}-\mathrm{H}), 7.68(1 \mathrm{H}, \mathrm{d}$, $J=8.7 \mathrm{~Hz}, \mathrm{Ar}-\mathrm{H}), 7.78(1 \mathrm{H}, \mathrm{d}, J=8.1 \mathrm{~Hz}, \mathrm{Ar}-\mathrm{H}), 7.90(1 \mathrm{H}$, $\mathrm{d}, J=8.4 \mathrm{~Hz}, \mathrm{Ar}-\mathrm{H}) .{ }^{13} \mathrm{C} \mathrm{NMR}\left(75.5 \mathrm{MHz}, \mathrm{CDCl}_{3}\right): \delta 26.84\left(2 \times \mathrm{CH}_{2}\right)$, $27.04\left(2 \times \mathrm{CH}_{2}\right), 29.81\left(\mathrm{ArCH} \mathrm{H}_{2} \mathrm{Ar}\right), 55.77\left(2 \times \mathrm{NCH}_{2}\right), 56.20\left(\mathrm{NCH}_{2}\right)$, $65.68\left(\mathrm{OCH}_{2}\right), 114.55(\mathrm{Ar}), 118.16(\mathrm{Ar}), 118.70(\mathrm{Ar}), 122.84(\mathrm{Ar})$, 123.31 ( $\mathrm{Ar}), 126.42$ ( $\mathrm{Ar}), 128.20(\mathrm{Ar}), 128.48(\mathrm{Ar}), 129.16(\mathrm{Ar})$, 129.21 (Ar), 129.89 (Ar), 132.68 (Ar), 133.72 (Ar), 151.77 (Ar), 156.91 (Ar). HRMS $m / z$ calculated for $\mathrm{C}_{25} \mathrm{H}_{29} \mathrm{NO}_{2}[\mathrm{M}+\mathrm{H}]^{+}$ 376.2271, observed $[\mathrm{M}+\mathrm{H}]^{+}$376. 2265.

\subsection{Molecular modeling}

Molecular mechanics and docking calculations were performed using the Molecular Operating Environment (Chemical Computing Group, Montreal, Canada). The ligand binding domain of the human estrogen receptor as deduced by protein X-ray crystallography (PDB ID: 1ERR) was retrieved from the Protein Data Bank (www.pdb.org) and prepared using the MMFF94 force field and charge groups. The model was reduced to include only one of two active sites, residues were physiologically protonated/deprotonated to $\mathrm{pH} 7.4$, and minimization with heavy atom tethers was used to remove potential bad contacts. The bound raloxifene was used as the primary template for docking of the molecules described in this paper [20]. In the docking methodology, up to 30 conformations were retained, triangle-matching was used for placement and London dG and/or Affinity dG were used for scoring. Binding modes presented graphically are representative of the highest-scored conformations.

\subsection{Methods for in-vitro biological assessments}

\subsubsection{Cytotoxicity activity}

The Molt4/C8, CEM and L1210 assays were conducted using a literature methodology [18]. Briefly, the tumor cells were seeded in 96-well microtiter plates and exposed to different concentrations of the test compounds. After 2 days (L1210) and 3 days (Molt4/ C8 and CEM), cell number was determined using a Particle counter (Coulter Z-1, Analis, Ghent, Belgium). The $\mathrm{IC}_{50}$ represents the compound concentration required to inhibit tumor cell proliferation by $50 \%$. MCF-7 cytotoxicity was measured using an MTS assay [27]. MCF-7 cells in DMEM containing 10\% heat-inactivated fetal bovine serum (FBS) were seeded into 24 -well plates $(30,000$ cells/ well). After treatment, the MTS assay (CellTiter 96 AQueous NonRadioactive Proliferation Assay; Fisher Scientific Ltd., Nepean, Ontario, Canada) was performed following the manufacturer's instruction. The cytostatic activity and cytotoxicity assays were performed in triplicate.

\subsubsection{NR peptide ER $\alpha$ ELISA assay}

The assay has 96 -well plate coated with a peptide that binds only to estrogen receptor that is in the ligand-activated conformation. Estrogen receptor captured through this interaction is then quantified through use of an estrogen receptor $\alpha$ antibody followed by colorimetric detection. This assay was performed to evaluate the antagonistic action of the most potent compound $\mathbf{3 g}$ according to the manufacturer's instructions. The kit contained a 96-well plate coated with peptide containing an ER $\alpha$ co-activator binding motif. All of the reagents were included in the kit. The vehicle control solution contained the MCF-7 nuclear extract as well as diluent buffer. Blank solution contained only diluent buffer. The $1 \mathrm{mM}$ stock solutions of tamoxifen (TEM, antagonist control) and estradiol (E2, agonist control) were diluted to $25 \mu \mathrm{M}$. Compound $\mathbf{3 b}$ was dissolved in water to form a $1 \mathrm{mM}$ stock solution. This stock solution was diluted to $25 \mu \mathrm{M}$ and $2.5 \mu \mathrm{M}$ for the ELISA assay. Nuclear extracts of $50 \mu \mathrm{g}$ per well was used, except for the agonist and antagonist controls, which had $15 \mu \mathrm{g}$ of nuclear extract per well as suggested by the manufacturer's instructions. The developing and stop solutions were added at the end of the procedure, allowing the amount of active co-activator-ER $\alpha$ complex to be quantified. The absorbance was measured on a Bio-Rad microplate reader. This assay was performed in duplicate at both concentrations.

\section{Acknowledgments}

The authors thank the Canadian Breast Cancer Foundation (Atlantic Chapter) and the K.U. Leuven (GOA no. 10/14) for grant support. VSP and YY thank the financial support provided by the University of Delhi under the DU-DST PURSE Grant. Lynn Thomas from Department of Biochemistry and Molecular Biology, Dalhousie University and Lizette van Berckelaer from Rega Institute of Medical Research, Katholieke Universiteit Leuven are thanked for technical supports.

\section{References}

[1] S. Nilsson, J.A. Gustafsson, Estrogen receptors: therapies targeted to receptor subtypes, Clin. Pharmacol. Ther. 89 (2011) 44-55.

[2] S.J. Howell, S.R.D. Johnston, A. Howell, The use of selective estrogen receptor modulators and selective estrogen receptor down-regulators in breast cancer, Best Pract. Res. Clin. Endocrinol. Metab. 18 (2004) 47-66.

[3] B.S. Katzenellenbogen, I. Choi, R. Delage-Mourroux, T.R. Ediger, P.G.V. Martini, M. Montano, J. Sun, K. Weis, J.A. Katzenellenbogen, Molecular mechanisms of estrogen action: selective ligands and receptor pharmacology, J. Steroid Biochem. Mol. Biol. 74 (2000) 279-285.

[4] W.-C. Park, V.C. Jordan, Selective estrogen receptor modulators (SERMS) and their roles in breast cancer prevention, Trends Mol. Med. 8 (2002) 82-88. 
[5] J.S. Lewis, V.C. Jordan, Selective estrogen receptor modulators (SERMs): mechanisms of anticarcinogenesis and drug resistance, Mutat. Res. 591 (2005) $247-263$.

[6] V.C. Jordan, Selective estrogen receptor modulation: concept and consequences in cancer, Cancer Cell 5 (2004) 207-213.

[7] D.L. Wickerham, J.P. Costantino, V.G. Vogel, W.M. Cronin, R.S. Cecchini, L.G. Ford, N. Wolmark, The use of tamoxifen and raloxifene for the prevention of breast cancer, Recent Results Cancer Res. 181 (2009) 113-119.

[8] L. Mosca, D. Grady, E. Barrett-Connor, P. Collins, N. Wenger, B.L. Abramson, A. Paganini-Hill, M.J. Geiger, S.A. Dowsett, M. Amewou-Atisso, M. Kornitzer, Effect of raloxifene on stroke and venous thromboembolism according to subgroups in postmenopausal women at increased risk of coronary heart disease, Stroke 40 (2009) 147-155.

[9] Z. Bai, R. Gust, Breast cancer, estrogen receptor and ligands, Arch. Pharm. 342 (2009) 133-149.

[10] C. Mukherjee, E.D. MacLean, T.S. Cameron, A. Jha, Enzyme-assisted kinetic resolution of novel 2-naphthol Mannich bases, J. Mol. Catal. B. Enzymatic 62 (2010) 46-53.

[11] A. Jha, N.K. Paul, S. Trikha, T.S. Cameron, Novel synthesis of 2-naphthol Mannich bases and their NMR behaviour, Can. J. Chem. 84 (2006) 843-853.

[12] J. Huang, G. Li, Z. Wu, Z. Song, Y. Zhou, L. Shuai, X. Weng, X. Zhou, G. Yang, Bisbenzimidazole to benzobisimidazole: from binding B-form duplex DNA to recognizing different modes of telomereG-quadruplex, Chem. Commun. (2009) 902-904.

[13] L. Nagarapu, Aneesa, A. Satyender, G. Chandana, R. Bantu, Synthesis and antimicrobial activity of novel analogs of trifenagrel, J. Heterocycl. Chem. 46 (2009) 195-200

[14] Q. Ji, J. Gao, J. Wang, C. Yang, X. Hui, X. Yan, X. Wu, Y. Xie, M.-W. Wang, Benzothieno[3,2-b]indole derivatives as potent selective estrogen receptor modulators, Bioorg. Med. Chem. Lett. 15 (2005) 2891-2893.

[15] X. Xu, J. Liu, L. Liang, H. Li, Y. Li, Iron-catalyzed regioselective hydroaryloxylation of $\mathrm{C}-\mathrm{C}$ triple bonds: an efficient synthesis of $2 \mathrm{H}-1$-benzopyran derivatives, Adv. Synth. Catal. 351 (2009) 2599-2604.

[16] J.R. Dimmock, A. Jha, P. Kumar, G.A. Zello, J.W. Quail, E.O. Oloo, J.J. Oucharek, M.K. Pasha, D. Seitz, R.K. Sharma, T.M. Allen, C.L. Santos, E.K. Manavathu, E. De Clercq, J. Balzarini, J.P. Stables, Cytotoxic 1,4-bis(2-oxo-1-cycloalkylmethylene) benzenes and related compounds, Eur. J. Med. Chem. 37 (2002) 35-44.
[17] J.R. Dimmock, P. Kumar, Anticancer and cytotoxic properties of Mannich bases, Curr. Med. Chem. 4 (1997)

[18] J. Balzarini, E. De Clercq, M.P. Mertes, D. Shugar, P.F. Torrence, 5-Substituted 2'-deoxyuridines: correlation between inhibition of tumor cell growth and inhibition of thymidine kinase and thymidylate synthetase, Biochem. Pharmacol. 31 (1982) 3673-3682.

[19] J.R. Dimmock, P. Kumar, A.J. Nazarali, N.L. Motaganahalli, T.P. Kowalchuk M.A. Beazely, J. Wilson Quail, E.O. Oloo, T.M. Allen, J. Szydlowski, E. DeClercq J. Balzarini, Cytotoxic 2,6-bis(arylidene)cyclohexanones and related compounds, Eur. J. Med. Chem. 35 (2000) 967-977.

[20] A.M. Brzozowski, A.C.W. Pike, Z. Dauter, R.E. Hubbard, T. Bonn, O. Engstrom, L. Ohman, G.L. Greene, J.-A. Gustafsson, M. Carlquist, Molecular basis of agonism and antagonism in the oestrogen receptor, Nature 389 (1997) 753-758.

[21] N.K. Paul, L. Dietrich, A. Jha, Convenient synthesis of 1-Arylmethyl-2naphthols, Synth. Commun. 37 (2007) 877-888

[22] H. Seeger, D. Diesing, B. Gückel, D. Wallwiener, A.O. Mueck, J. Huober, Effect of tamoxifen and 2-methoxyestradiol alone and in combination on human breast cancer cell proliferation, J. Steroid Biochem. Mol. Biol. 84 (2003) 255-257.

[23] T. Paimela, J.M.T. Hyttinen, J. Viiri, T. Ryhänen, M.K. Karvonen, M. Unkila, H. Uusitalo, A. Salminen, K. Kaarniranta, Influence of selective estrogen receptor modulators on interleukin-6 expression in human retinal pigment epithelial cells (ARPE-19), Eur. J. Pharmacol. 640 (2010) 219-225.

[24] I. Paternotte, H.J. Fan, P. Scrève, M. Claesen, P.M. Tulkens, E. Sonveaux, Syntheses and hydrolysis of basic and dibasic ampicillin esters tailored for intracellular accumulation, Bioorg. Med. Chem. 9 (2001) 493-502.

[25] A. Ryckebusch, D. Garcin, A. Lansiaux, J.-F. Goossens, B. Baldeyrou, R. Houssin, C. Bailly, J.-P. Hénichart, Synthesis, cytotoxicity, DNA interaction, and topoisomerase II inhibition properties of novel indeno[2,1-c]quinolin-7-one and indeno[1,2-c]isoquinolin-5,11-dione derivatives, J. Med. Chem. 51 (2008) 3617-3629.

[26] R. Laudien, I. Yoshida, T. Nagamura, Synthesis and photophysical properties of porphyrins containing viologen units for ultrafast molecular photonics, J. Chem. Soc. Perkin Trans. 2 (2002) 1772-1777.

[27] S.A. Abdelmagid, C.K.L. Too, Prolactin and estrogen up-regulate carboxypeptidase-D to promote nitric oxide production and survival of MCF-7 breast cancer cells, Endocrinology 149 (2008) 4821-4828. 